\title{
Organocatalytic Highly Enantioselective Nitroaldol Reaction of $\alpha$-Ketophosphonates and Nitromethane
}

\author{
Tanmay Mandal, Sampak Samanta, and Cong-Gui Zhao* \\ Department of Chemistry, University of Texas at San Antonio
}

Supporting Information

TABLE OF CONTENTS

Experimental Procedures ………………………….............................................

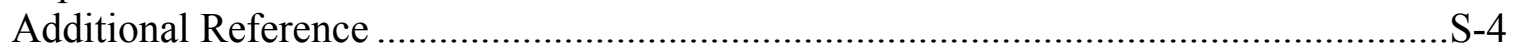

NMR Spectra of New Compounds …………………..........................................

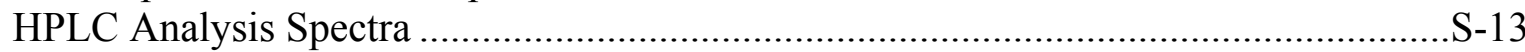

\section{Experimental Procedures}

\section{General}

${ }^{1} \mathrm{H}$ and ${ }^{13} \mathrm{C}$ NMR spectra were obtained on a Varian INOVA $500 \mathrm{MHz}$ spectrometer. HPLC analysis was performed on a Shimadzu instrument with LC-10AT pump and SPD10AV UV-Vis detector. Chiralcel and Chiralpak HPLC columns were purchased from Daicel Chemical Industry, Ltd. Except for ethyl benzoylphosphonate (4a), which was purchased from Aldrich and used as received, $\alpha$-ketophosphonates, ${ }^{13}$ as well as the catalysts, cupreine (2) ${ }^{11 \mathrm{a}, 12}$ and 9-O-benzylcupreine (3), ${ }^{11 \mathrm{a}, 12}$ were prepared by using the reported procedures. THF was freshly distilled over potassium. Other reagents and solvents were purchased from either Aldrich or Acros and used as received.

General experimental procedure for the enantioselective nitroaldol reaction of $\alpha$-ketophosphonates and nitromethane 


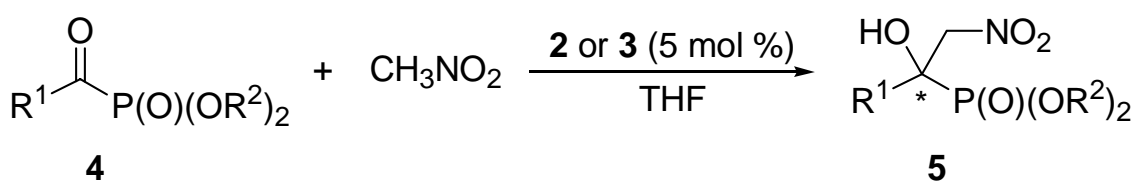

To a stirred solution of the $\alpha$-ketophosphonate $(0.25 \mathrm{mmol})$ and nitromethane $(0.2 \mathrm{~mL})$ in dry THF $(0.2 \mathrm{~mL})$ was added catalyst $2(3.9 \mathrm{mg}, 0.0125 \mathrm{mmol}, 5 \mathrm{~mol} \%)$ at $0{ }^{\circ} \mathrm{C}$ and the reaction mixture was allowed to stir for the time as specified in Table 2 (monitored by TLC). Afterwards the reaction mixture was quenched by adding a few drops of saturated ammonium chloride solution. The reaction mixture was then extracted with ethyl acetate ( $2 \times 10 \mathrm{~mL}$ ), washed with brine $(2 \mathrm{~mL})$ and dried over $\mathrm{MgSO}_{4}$. The crude product, obtained after evaporation of the solvent, was purified by column chromatography (eluting with EtOAc/hexane) to provide the pure desired product.

In the case of catalyst $\mathbf{3}$, all the reactions were carried out in a similar manner; except for the reaction temperature was room temperature.

Most of the products are known compounds, ${ }^{7}$ except for the following:

\section{Diethyl [1-(4-Bromophenyl)-1-hydroxy-2-nitroethyl]phosphonate (5k)}

White needles, mp 110-112 ${ }^{\circ} \mathrm{C}$; IR (neat): $v_{\max } 3173,1558,1486,1420 \mathrm{~cm}^{-1} ;{ }^{1} \mathrm{H}$ NMR (500 MHz, $\left.\mathrm{CDCl}_{3}\right) \delta 1.20(\mathrm{t}, J=6.5 \mathrm{~Hz}, 3 \mathrm{H}), 1.30(\mathrm{t}, J=6.5 \mathrm{~Hz}, 3 \mathrm{H}), 3.85-3.90$ $(\mathrm{m}, 1 \mathrm{H}), 3.98-4.01(\mathrm{~m}, 1 \mathrm{H}), 4.14-4.18(\mathrm{~m}, 2 \mathrm{H}), 4.52-4.55$ ( br s, 1H), $5.02\left(\mathrm{dd}, J_{1}=4.0\right.$, $\left.J_{2}=13.5 \mathrm{~Hz}, 1 \mathrm{H}\right), 5.12\left(\mathrm{dd}, J_{1}=7.0, J_{2}=12.5 \mathrm{~Hz}, 1 \mathrm{H}\right), 7.48-7.54(\mathrm{~m}, 4 \mathrm{H}) ;{ }^{13} \mathrm{C} \mathrm{NMR}$ $\left(125 \mathrm{MHz}, \mathrm{CDCl}_{3}\right) \delta 16.5$ (t), 64.7 (d), $64.9(\mathrm{~d}), 75.40\left(\mathrm{~d}, J_{\mathrm{CP}}=163.6 \mathrm{~Hz}\right), 79.9(\mathrm{~d})$, 123.3, 128.1 (d), 131.9 (d), 134.6. Anal. Calcd. for $\mathrm{C}_{12} \mathrm{H}_{17} \mathrm{BrNO}_{6} \mathrm{P}: \mathrm{C}, 37.72 ; \mathrm{H}, 4.48$; N, 3.67. Found: C, 37.85; H, 4.60; N, 3.68.

\section{Diethyl [1-Hydroxy-1-(4-methoxyphenyl)-2-nitroethyl]phosphonate (5n)}

White needles, mp $92-94{ }^{\circ} \mathrm{C}$; IR (neat): $v_{\max } 3200,1604,1550,1510,1443,1421$, $1392 \mathrm{~cm}^{-1}$; ${ }^{1} \mathrm{H}$ NMR $\left(500 \mathrm{MHz}, \mathrm{CDCl}_{3}\right) \delta 1.17(\mathrm{t}, J=7.00 \mathrm{~Hz}, 3 \mathrm{H}), 1.31(\mathrm{t}, J=7.00 \mathrm{~Hz}$, $3 \mathrm{H}), 3.76-3.86(\mathrm{~m}, 4 \mathrm{H}), 3.92-4.00(\mathrm{~m}, 1 \mathrm{H}), 4.11-4.19(\mathrm{~m}, 2 \mathrm{H}), 4.35 \& 4.38(2 \mathrm{~s}, 1 \mathrm{H})$, $\left.5.017\left(\mathrm{dd}, J_{1}=4.0, J_{2}=13.5 \mathrm{~Hz}, 1 \mathrm{H}\right), 5.15\left(\mathrm{dd}, J_{1}=7.5, J_{2}=13.5 \mathrm{~Hz}, 1 \mathrm{H}\right), 1 \mathrm{H}\right), 6.92(\mathrm{~d}$, $J=8.5 \mathrm{~Hz}, 2 \mathrm{H}), 7.52\left(\mathrm{dd}, J_{1}=2.5, J_{2}=8.5 \mathrm{~Hz}\right) ;{ }^{13} \mathrm{C} \mathrm{NMR}\left(125 \mathrm{MHz}, \mathrm{CDCl}_{3}\right) \delta 16.5(\mathrm{t})$, 
55.5, 64.3 (d), 64.8 (d), 75.4 (d, $\left.J_{\mathrm{CP}}=166.0 \mathrm{~Hz}\right), 80.1$ (d), $114.2(\mathrm{~d}), 127.0,127.6$ (d), 160.1 (d). Anal. Calcd. for $\mathrm{C}_{13} \mathrm{H}_{20} \mathrm{NO}_{7} \mathrm{P}: \mathrm{C}, 46.85 ; \mathrm{H}, 6.05 ; \mathrm{N}, 4.20$. Found: $\mathrm{C}, 47.10 ; \mathrm{H}$, $6.21 ; \mathrm{N}, 4.20$.

\section{Diethyl [1-Hydroxy-2-nitro-1-(thiophen-2-yl)ethyl]phosphonate (50)}

White needles, mp $76-78^{\circ} \mathrm{C}$; IR (neat): $v_{\max } 3210,1561,1410,1390 \mathrm{~cm}^{-1}$; ${ }^{1} \mathrm{H}$ NMR $\left(500 \mathrm{MHz}, \mathrm{CDCl}_{3}\right) \delta 1.24(\mathrm{t}, J=7.00 \mathrm{~Hz}, 3 \mathrm{H}), 1.32$ ( t $\left., J=7.00 \mathrm{~Hz}, 3 \mathrm{H}\right), 3.92-3.99$ (m, $1 \mathrm{H})$, 4.03-4.09 (m, 1H), 4.17-4.22 (m, 2H), 5.02-5.02 (m, 3H), 7.01-7.03 (m, 1H), 7.14$7.15(\mathrm{~m}, 1 \mathrm{H}), 7.33-7.34(\mathrm{~m}, 1 \mathrm{H}) ;{ }^{13} \mathrm{C} \mathrm{NMR}\left(125 \mathrm{MHz}, \mathrm{CDCl}_{3}\right) \delta 16.5$ (q), $64.8(\mathrm{~d}), 65.1$ (d), $74.7\left(\mathrm{~d}, J_{\mathrm{CP}}=169.7 \mathrm{~Hz}\right), 80.3$ (d), 125.9 (d), 126.8 (d), 127.6 (d), 139.6 (d). Anal. Calcd. for $\mathrm{C}_{10} \mathrm{H}_{16} \mathrm{NO}_{6} \mathrm{PS}$ : C, 38.83; H, 5.21; N, 4.53. Found: C, 39.04; H, 5.31; N, 4.57.

\section{Experimental procedure for the reduction of the nitroaldol product $5 \mathrm{c}$ to a $\beta$-amino-} $\alpha$-hydroxyphosphonate

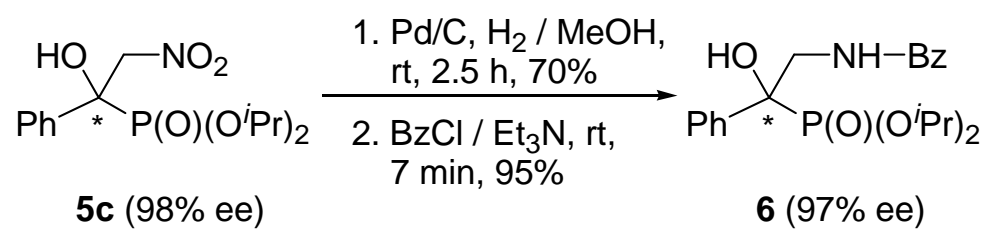

To a solution of diisopropyl [1-hydroxy-2-nitro-1-phenylethyl]phosphonate (5c, $331 \mathrm{mg}$, $1.0 \mathrm{mmol}, 98 \%$ ee) in $10 \mathrm{~mL}$ of methanol was added $60 \mathrm{mg}$ of palladium over activated charcoal $(10 \% \mathrm{w} / \mathrm{w})$. The mixture was stirred under hydrogen atmosphere (1 atm) until the completion of the reduction $(2.5 \mathrm{~h}$, monitored by TLC). The reaction mixture was passed through a plug of Celite and washed with methanol $(10 \mathrm{~mL})$. An oily product was obtained after methanol was evaporated ( $211 \mathrm{mg}, 70 \%$ yield).

To determine the ee value of this product, it was directly protected with a benzoyl group without further purification: the reduced product was dissolved in $\mathrm{CH}_{2} \mathrm{Cl}_{2}(5 \mathrm{~mL})$, and triethyl amine $(85 \mathrm{mg}, 0.84 \mathrm{mmol})$ and benzoyl chloride $(108 \mathrm{mg}, 0.77 \mathrm{mmol})$ were added at the room temperature with stirring. The reaction completed after $7 \mathrm{~min}$ as indicated by TLC analysis. Afterwards the reaction mixture was extracted with ethyl acetate $(5 \mathrm{ml} \times 3)$. The combined extracts were washed with brine $(5 \mathrm{ml} \times 2)$ and dried 
over $\mathrm{MgSO}_{4}$. Evaporation of the solvent yielded the crude product, which was purified by column chromatography to give the pure compound 6 ( $270 \mathrm{mg}$, 95\% yield). This product was characterized by its ${ }^{1} \mathrm{H}$ and ${ }^{13} \mathrm{C}$ NMR spectroscopic data: colorless needles solid, mp 125-127 ${ }^{\circ} \mathrm{C}$; IR (neat) v 3277, 1687, 1637, 1576, 1559, 1475, 1459, $1318 \mathrm{~cm}^{-1} ;{ }^{1} \mathrm{H}$ NMR ( $\left.\mathrm{CDCl}_{3}, 500 \mathrm{MHz}\right) \delta 0.91(\mathrm{~d}, J=6.0 \mathrm{~Hz}, 3 \mathrm{H}), 1.15(\mathrm{~d}, J=6.00 \mathrm{~Hz}, 3 \mathrm{H}), 1.19(\mathrm{~d}, J=$ $6.00 \mathrm{~Hz}, 3 \mathrm{H}$ ), 1.24 ( d, $J=6.00 \mathrm{~Hz}, 3 \mathrm{H}), 4.09-4.20$ (m, 2H), 4.39-4.43 (m, 1H), 4.68-4.72 (m, 1H), 5.4 ( brs, $1 \mathrm{H}), 7.27-7.55(\mathrm{~m}, 7 \mathrm{H}), 7.73-7.78(\mathrm{~m}, 4 \mathrm{H}) \mathrm{ppm} ;{ }^{13} \mathrm{C} \mathrm{NMR}\left(\mathrm{CDCl}_{3}\right.$, $125 \mathrm{MHz}) \delta 23.3$ (d), 24.0 (d), 24.1 (d), 24.4 (d), 48.9 (d), 72.5 (d), 72.8 (d), 77.5 (d, $J_{\mathrm{cp}}=$ $163.00 \mathrm{~Hz}), 126.7$ (d), 127.5 , 127.8 (d), 128.2 (d), 128.6, 131.9, 133.9, 138.7, 169.9 ppm.

The ee value of compound 6 was determined to $97 \%$ by HPLC analysis using a Chiralpak AD-H column.

\section{Additional Reference}

13. Baccolini, G.; Faggiano, M.; Todesco, P. E. J. Chem. Soc., Perkin Trans. 1 1979, 2329-2333. 


\section{Archlve directory: /home/samanta/vnmrsys/data}

Archlve directory:

Pulse Sequence: s2pul

Solvent: $\operatorname{CDC} 13$

Amblent temperature
INOVA-500 "I nova500"

Relax. delay $1.000 \mathrm{sec}$

Pulse 45.0 degrees

Acq $t 1 \mathrm{me} 1.892 \mathrm{sec}$

8 repetitions

OBSERVE H1, $499.6847200 \mathrm{MHz}$

DATA PROCESSING

FT s1ze 32768
Total time $0 \mathrm{~m} 1 \mathrm{n}, 23 \mathrm{sec}$

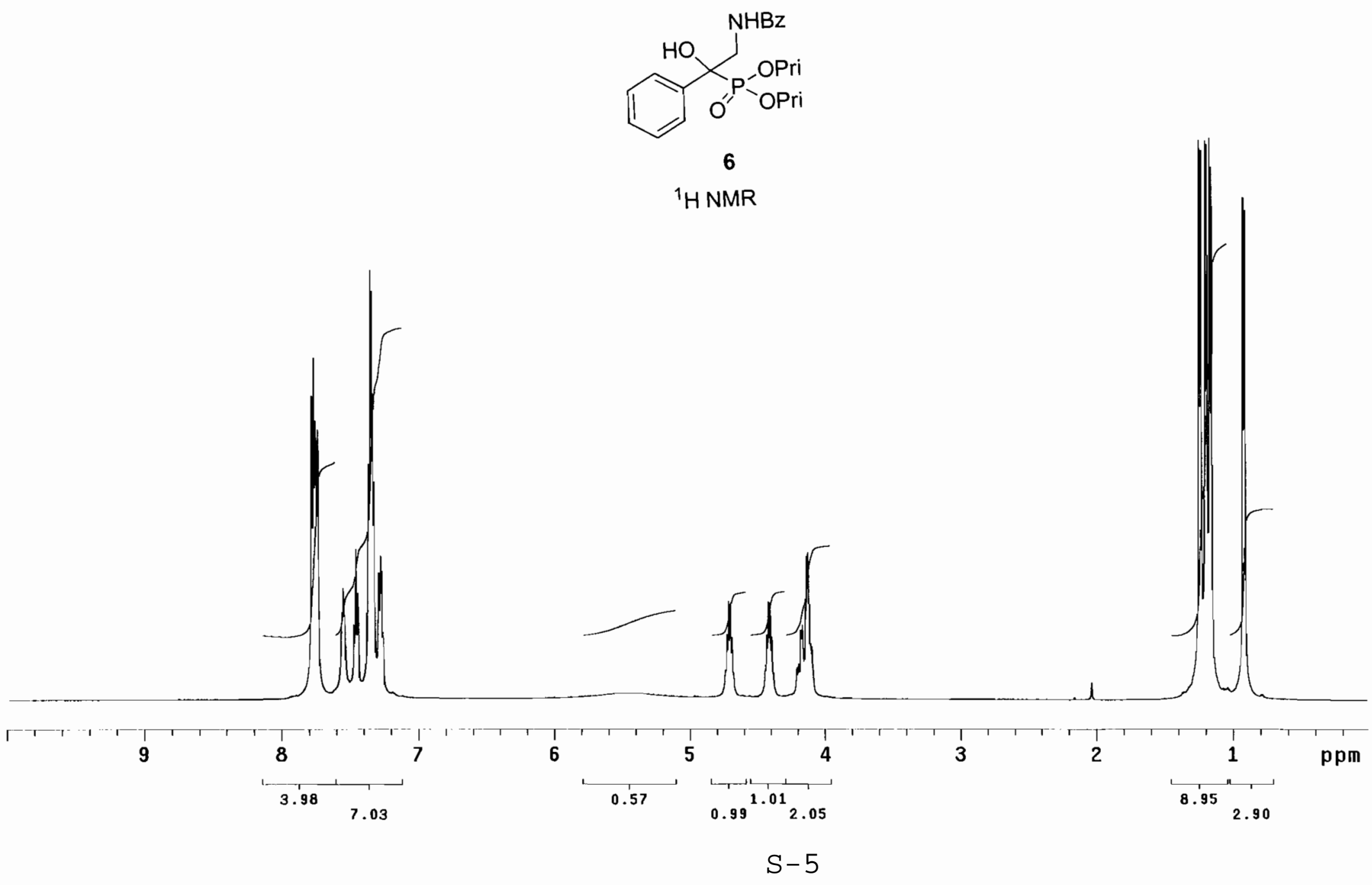


STANDARD CARBON PARAMETERS

Archive directory: /home/samanta/Unmrsys/data Sample directory

Pulse Sequence: s2pul

Solvent: $\mathrm{CDC}_{13}$

Ambient temperature

USer: $1-14-87$
INOVA-500 "Inova500"

Relax. delay $1.000 \mathrm{sec}$

Acq. time 1.300 sec

Width $31409.5 \mathrm{H}$

Power $36 \mathrm{~dB}$

COnt

DATA PROCESSING

Line broadening $0.5 \mathrm{~Hz}$

Pulse 45.0 degrees

OBSERYE C13, 125.6457928 MHZ

DECOUPLE H1, 499.6872185 MH

Total time $38 \mathrm{~min}, 29 \mathrm{sec}$

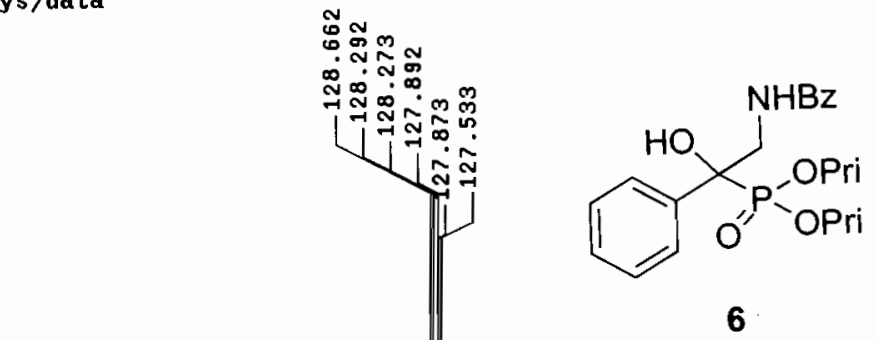

${ }^{13}$ C NMR
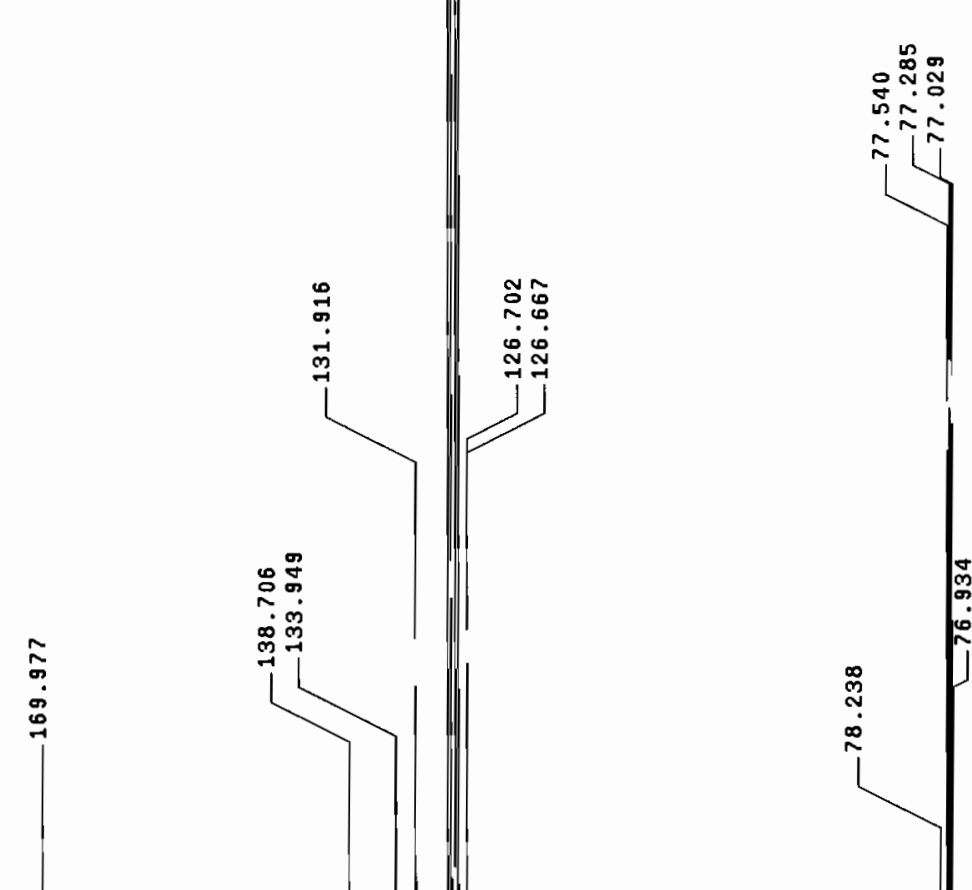

220

200

180

160

140

120

100 S- 6$$
80
$$

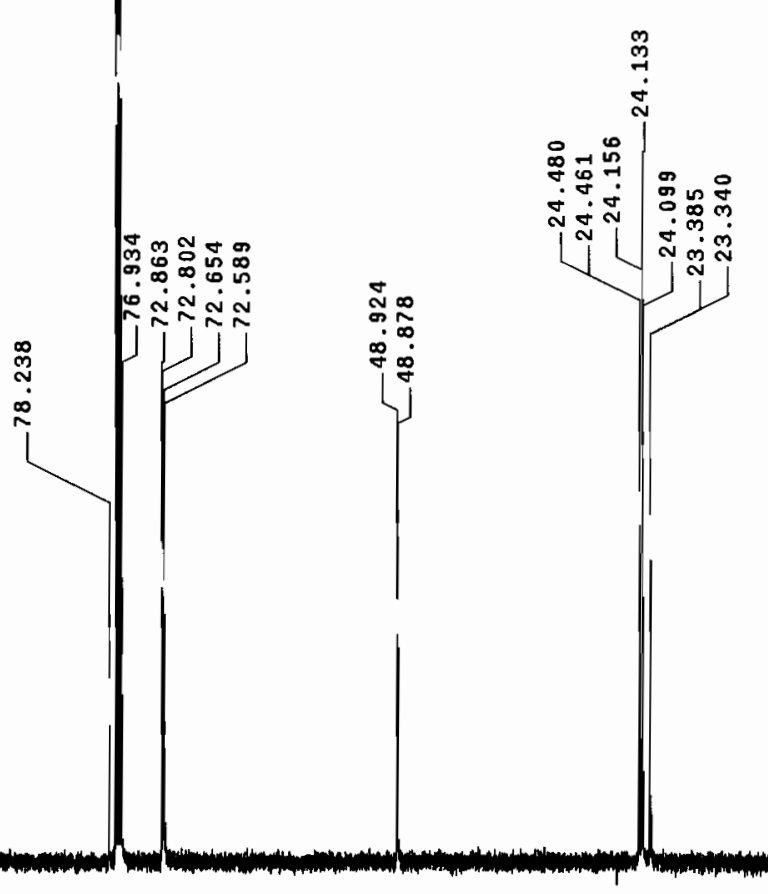

60

40

20

0 
STANDARD PROTON PARAMETERS

VII-SS-122

Archlve directory: /home/samanta/unmrsys/data

Pulse Sequence: s2pul

Solvent: $\operatorname{CDC}_{13}$

erature

INOVA-500 "InOVa500"

Relax. de lay $1.000 \mathrm{sec}$

Pulse 45.0 degrees

Acq. t $1 \mathrm{me} 1.892 \mathrm{sec}$

Width $7995.2 \mathrm{~Hz}$

OBSERVE H1 499.6847200 MHZ

DATA PROCESSING

FT S IZE 32768

Total time $0 \mathrm{~min}, 58 \mathrm{sec}$
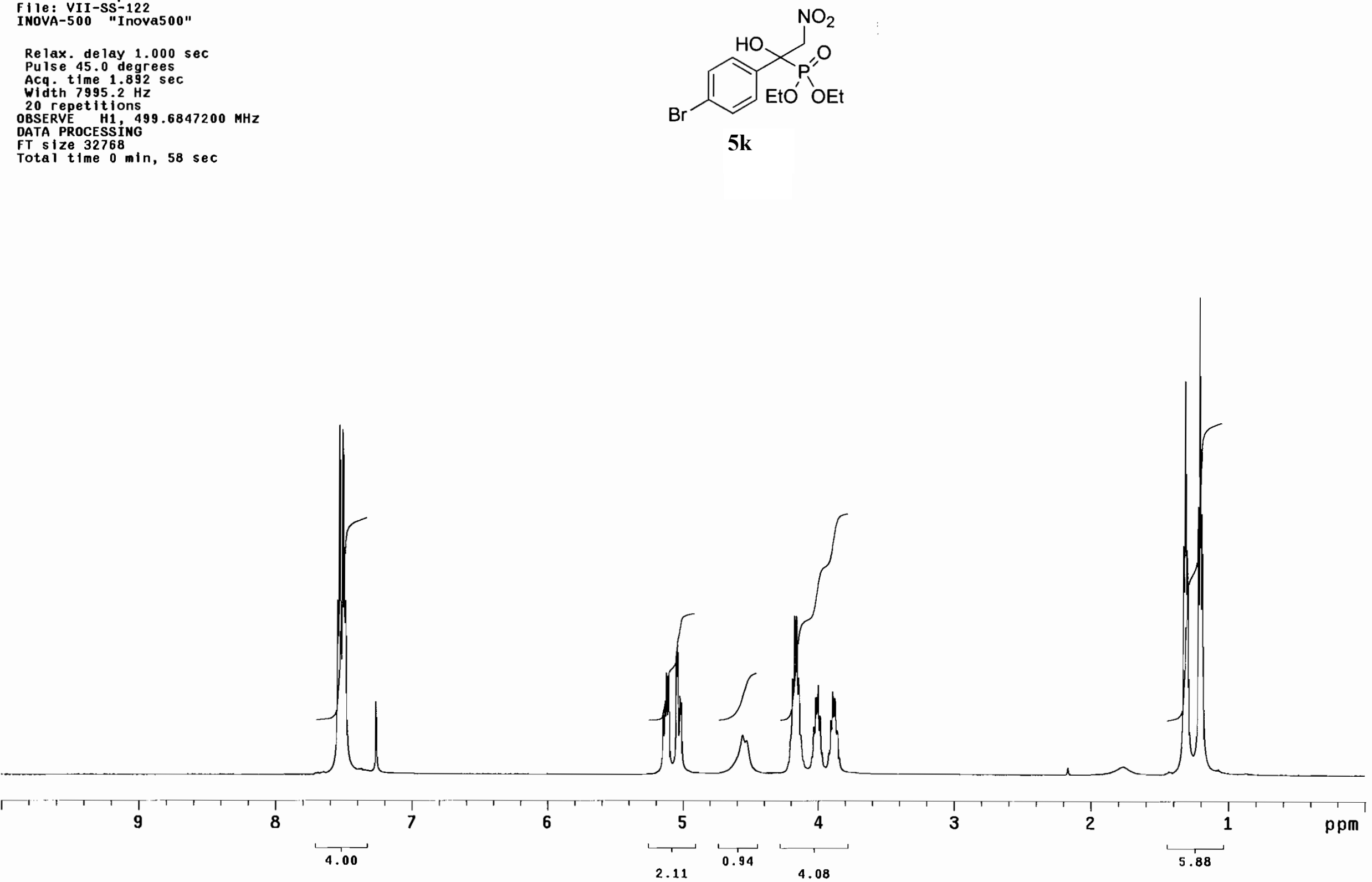
Pulse Sequence: s2pur

Solvent: $\operatorname{CDC} 13$

Amb1ent temperature

User: 1-14-87

F1 T: VII-SS-122C
INOVA-500 "InOVa500"

Re lax. de lay $1.000 \mathrm{sec}$

Pulse 45.0 degrees

Acq. t1me $1.300 \mathrm{~s}$

1000 repetitions

OBSERVE C13, 125.6457928 MH

DECOUPLE H1, 499.6872185 MHZ

Power 36 dB

WAL TZ-16 ly on

DATA PROCESSING

Line broadening $0.5 \mathrm{~Hz}$

FT s1ze 131072

Total time $38 \mathrm{~min}, 29 \mathrm{sec}$

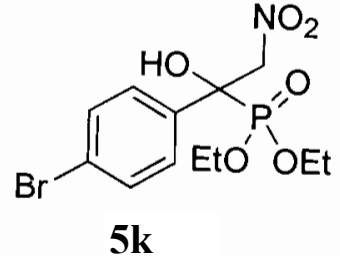

$5 \mathbf{k}$

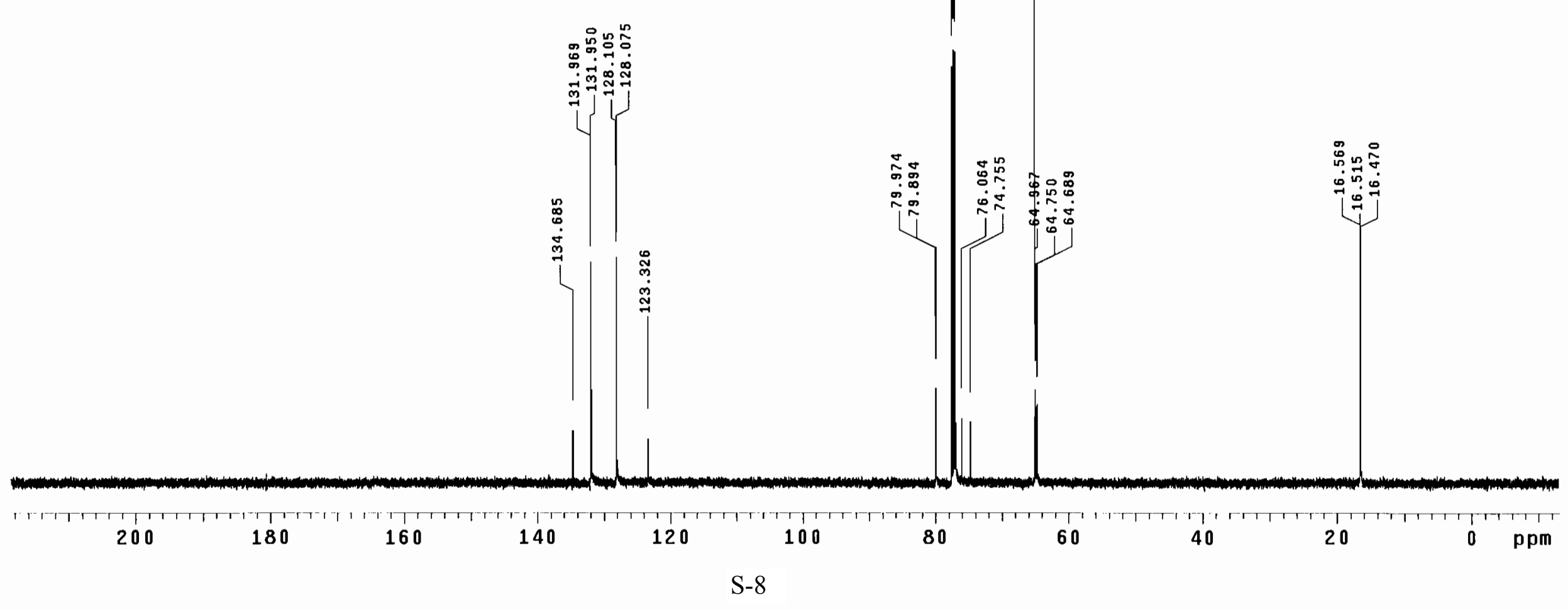


STANDARD PROTON PARAMETERS

VII-SS-129

Archive directory: /home/samanta/vnmrsys/data

Sample directory:

Pulse Sequence: s2pul

Solvent: $\operatorname{CDC13}$

Ambient temperature

INOVA-500 "InOVa500"

Relax. delay $1.000 \mathrm{sec}$

Pulse 45.0 degrees

Acq t tme $1.892 \mathrm{sec}$

8 repetitions

OBSERVE H1, 499.6847200 MHz

DATA PROCESSING

Total time $0 \mathrm{~m} / \mathrm{n}, 23 \mathrm{sec}$

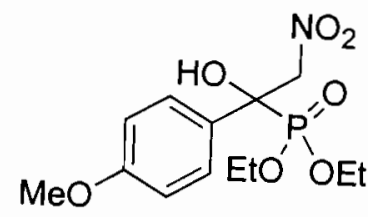

5n

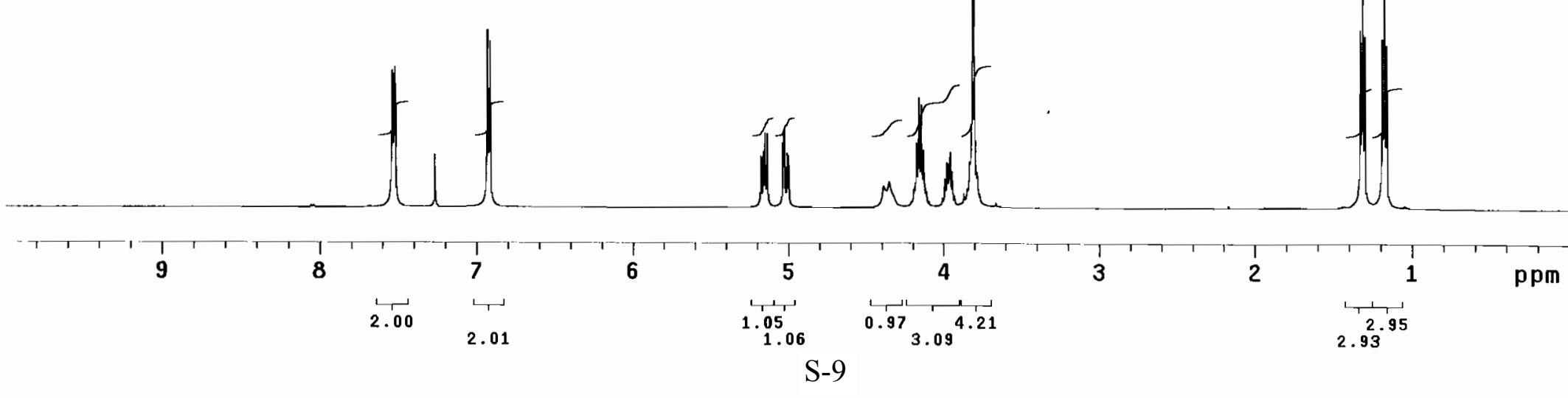


STANDARD CARBON PARAMETERS VII-SS1-129C

Archive directory: /home/samanta/unmrsys/data

Pulse Sequence: s2pur

Solvent: $\operatorname{CoC} 13$

Ambient temperature

User: 1-14-87

File: VII-SS-129c

Relax delay 1.000 sec

Pulse 450 degrees

Acg. time 1.300 sec

Width $31409.5 \mathrm{~Hz}$

1000 repetitions

OBSERVE C13, 125.6457928 MHZ

DECUPLE HI, A99.6872185 $\mathrm{MHZ}$

$$
\begin{aligned}
& \text { Power } 36 \mathrm{~dB} \\
& \text { cont inuous Iy }
\end{aligned}
$$

WALTZ-16 modulated

DATA PROCESSING

Line broadening $0.5 \mathrm{~Hz}$

Total time $38 \mathrm{~min}, 29 \mathrm{sec}$

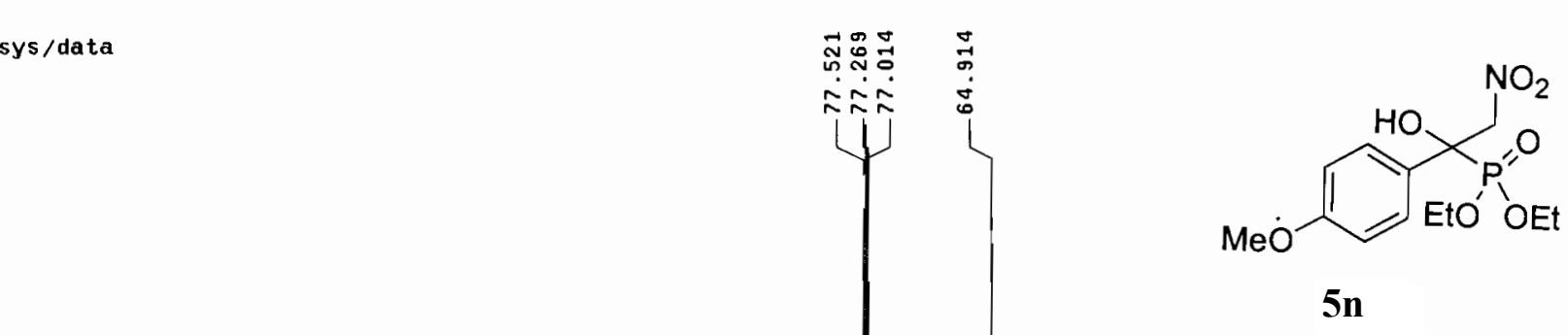

-
$\begin{array}{rllllll}1 & 1 & 1 & 1 & 1 & 1 & 1 \\ 2 & 0 & 0\end{array}$
180
160
140
120
100
60
40
20
0 ppm

S-10 
STANDARD PROTON PARAMETERS

VII-SS-131

Archive directory: /home/samanta/vnmrsys/data

1 e directory:

Pulse Sequence: s2pul

Solvent: $\operatorname{CoC} 13$

Ambient temperature

File: VII-SS-131
INOVA-500 "InOVa500"

Re lax. de lay $1.000 \mathrm{sec}$

Pulse 45.0 degrees

Acq. t t me $1.892 \mathrm{sec}$

Width $7995.2 \mathrm{~Hz}$

OBSERVE H1, 499.6847200 MHZ

DATA PROCESSINC

TOt 1 ize 32768 in, 2350

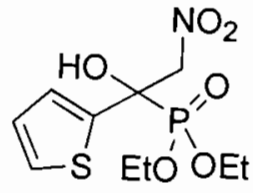

50

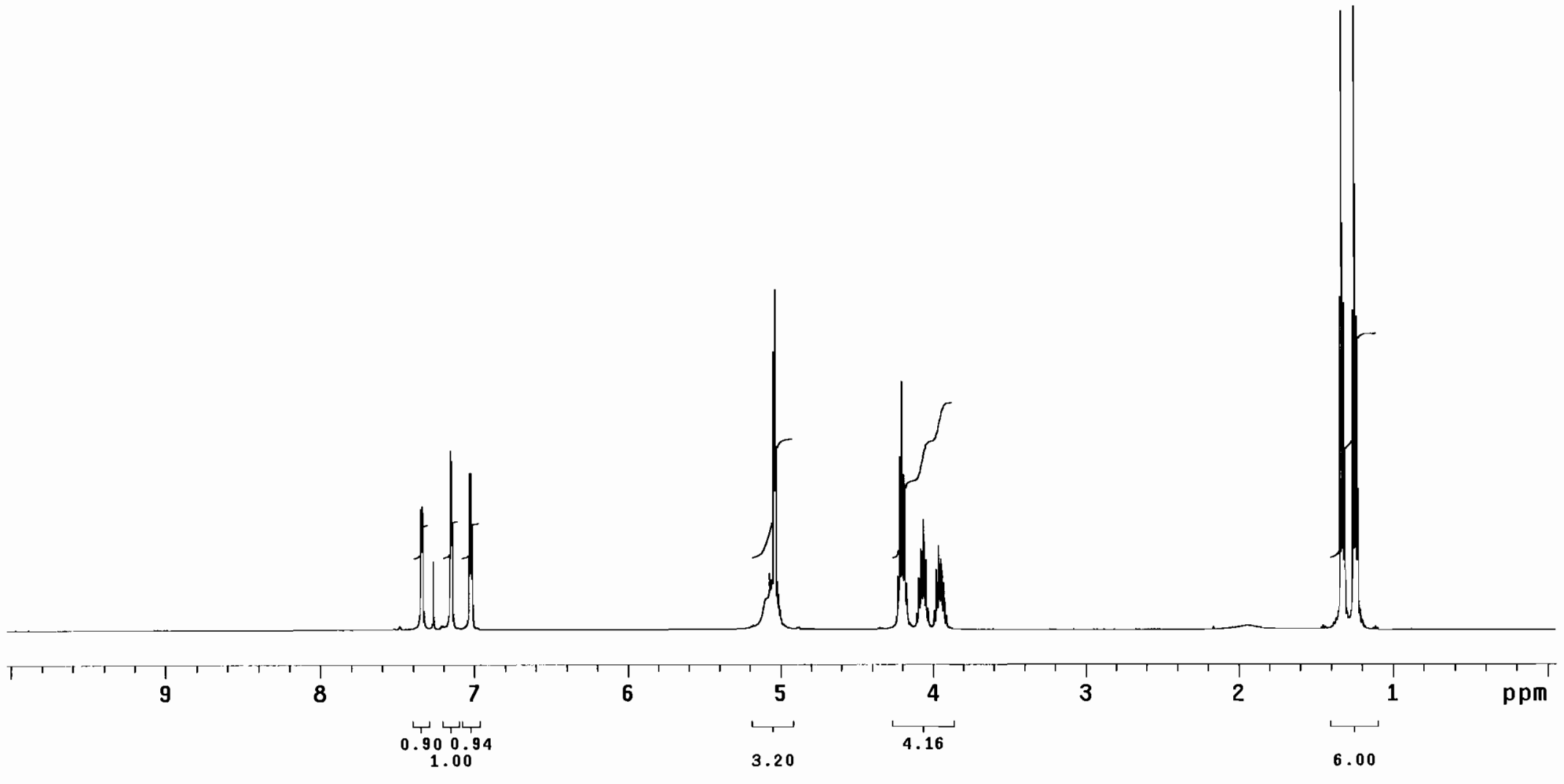

S-11 
STANDARD CARBON PARAMETERS

VII-SS-131C

Archlve directory: /home/samanta/unmrsys/data

Samp le directory:

Pulse Sequence: s2pul

Solvent: $\operatorname{CDC} 13$

Amblent temperature

Ile: VII-SS-131C

INOVA-500 "InOVa500"

Relax. de lay $1.000 \mathrm{sec}$

Pulse 45.0 degrees

Acq. $t$ tme $1.300 \mathrm{sec}$

Widh 31409.5 Hz

OBSERYE C13, 125.6457928 MHZ

DECOUPLE H1, 499.6872185 MHZ

Power 36 dB

continuous ly on

WALA PROCESSING

Line broadening $0.5 \mathrm{~Hz}$

Tize 131072

total time $1 \mathrm{hr}, 36 \mathrm{~m} 1 \mathrm{n}, 13 \mathrm{sec}$

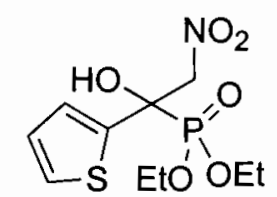

50

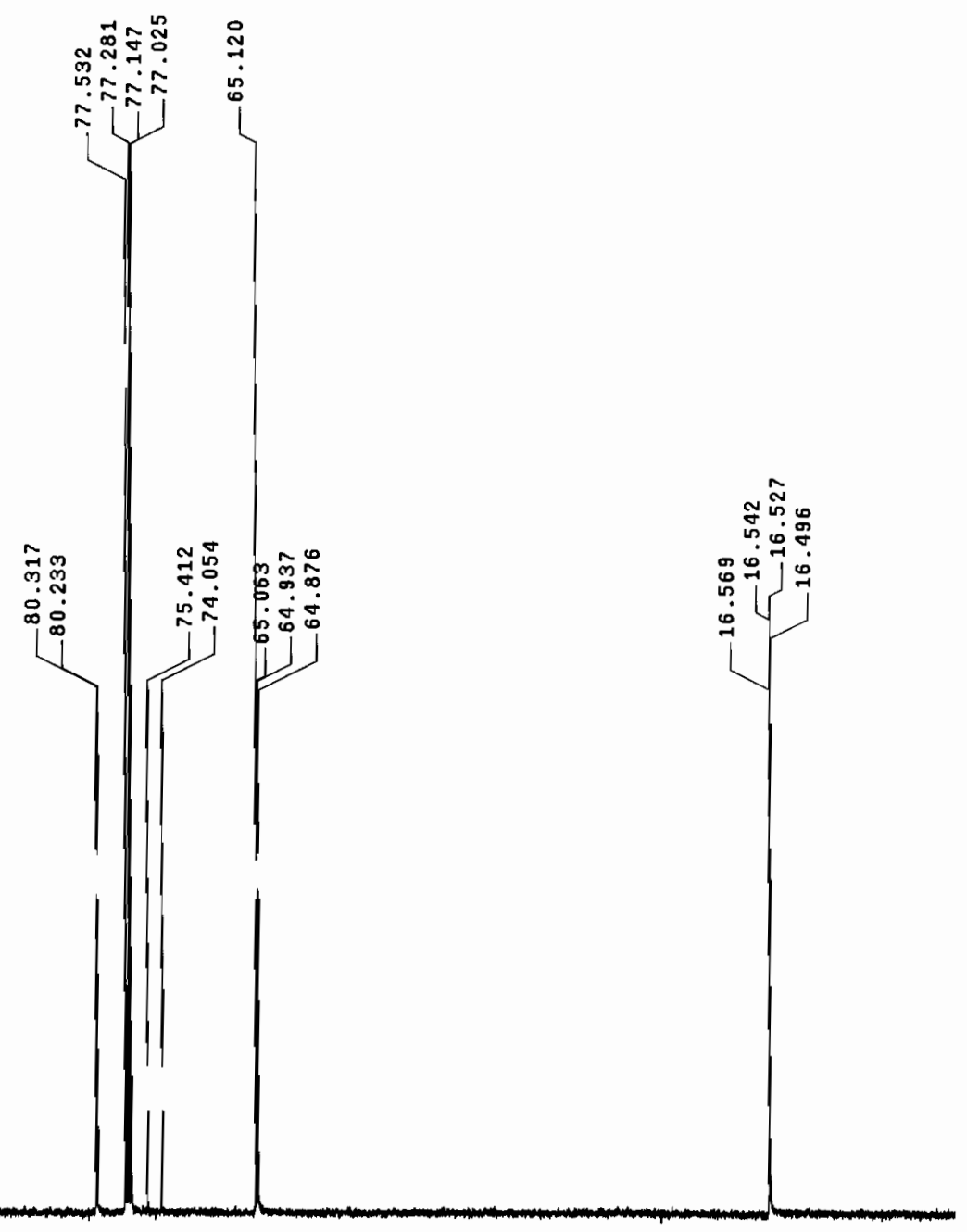




\section{Area \% Report}

Method Name: C: $\backslash$ EZStart $\backslash$ Projects $\backslash$ Default $\backslash$ Methods $\backslash$ AS.met

Data: C: $\backslash$ EZStart $\backslash$ Projects $\backslash$ Default $\backslash$ Data $\backslash I-T M-1$

User: $\quad$ System

Acquired: $\quad$ 10/23/2006 4:00:28 PM

Printed: $\quad 12 / 13 / 2006$ 2:25:50 PM

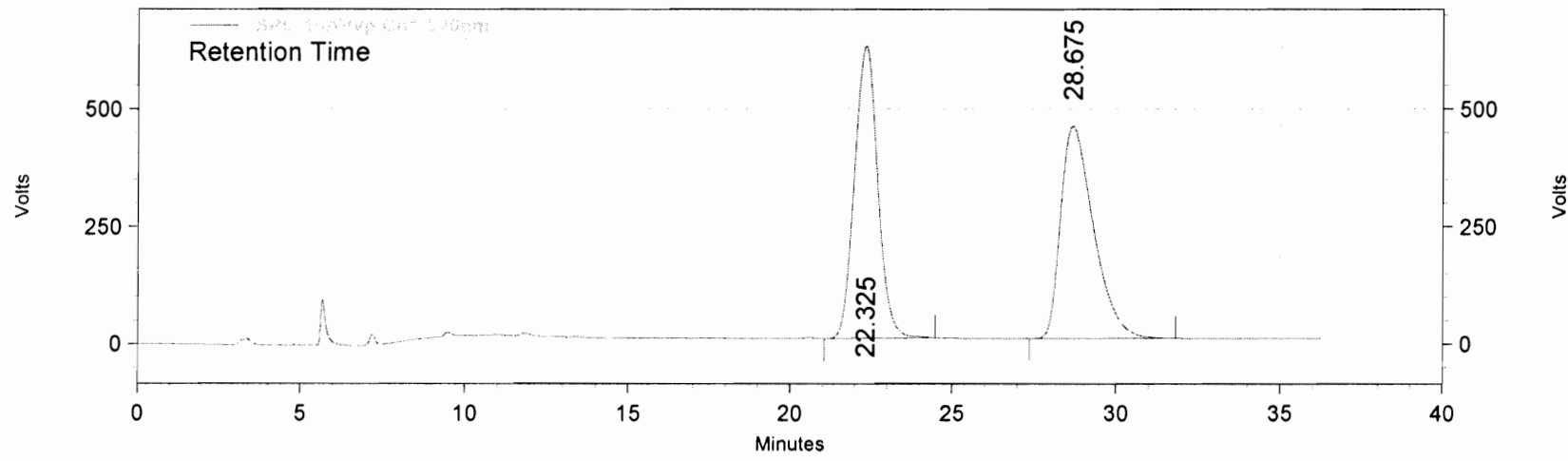

\section{SPD-10AVvp}

Ch1-220nm

Results

Retention Time 22.325

Area Area \%

Height Height \%

28.675

31039191

49.63

50.37

621121

450847

42.06

\begin{tabular}{|r|r|r|r|r|}
\hline Totals & 62536778 & 100.00 & 1071968 & 100.00 \\
\hline
\end{tabular}<smiles>CCOP(=O)(OCC)C(O)(C[N+](=O)[O-])c1ccccc1</smiles>

(5a, Racemic) 


\section{Area \% Report}

Method Name: C: $\backslash$ EZStart $\backslash$ Projects $\backslash$ Default $\backslash$ Methods $\backslash$ AS.met

Data:

User:

C: $\backslash$ EZStart $\backslash$ Projects $\backslash$ Default $\backslash$ Data $\backslash I-T M-105 O J$

Acquired: $\quad$ 12/4/2006 11:14:52 AM

Printed: $\quad 12 / 13 / 2006$ 10:30:06 AM

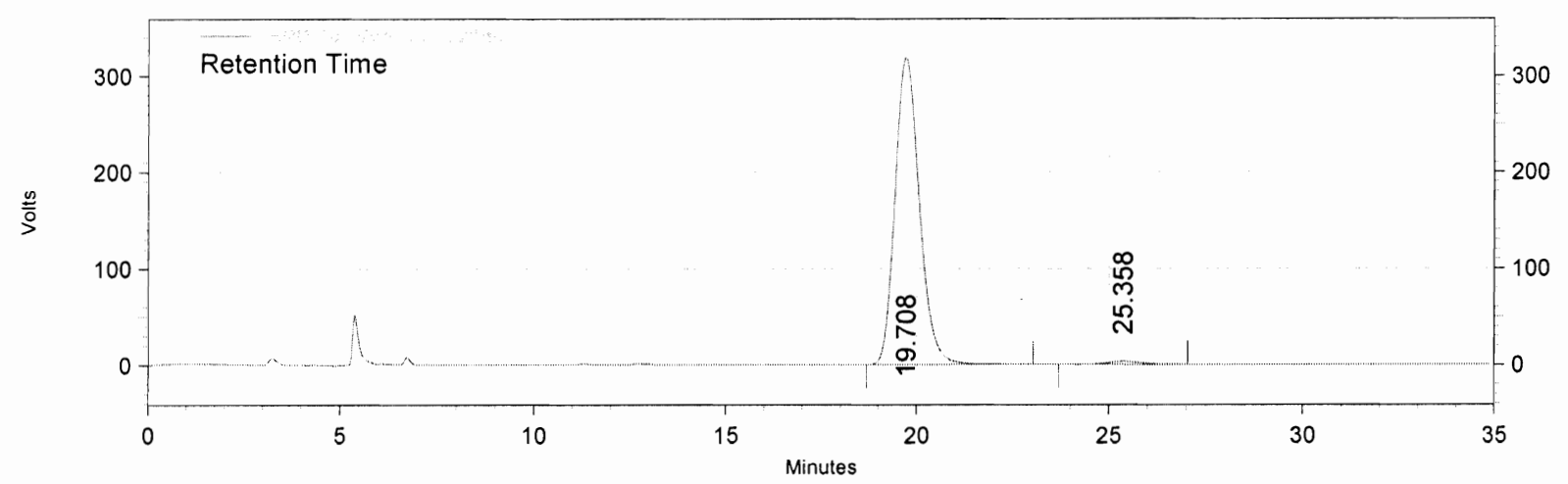

SPD-10AVvp

Ch1-220nm

Results

Retention Time

Area

Area \%

Height

Height \%

19.708

14108786

98.79

318799

99.08

25.358

172407

1.21

2950

0.92

\begin{tabular}{|r|r|r|r|r|}
\hline Totals & 14281193 & 100.00 & 321749 & 100.00 \\
\hline
\end{tabular}<smiles>CCOP(=O)(OCC)[C@@](O)(C[N+](=O)[O-])c1ccccc1</smiles>

(5a, $98 \%$ ee)

catalyst 3 


\section{Area \% Report}

Method Name: C: $\backslash$ EZStart $\backslash$ Projects $\backslash$ Default $\backslash$ Methods $\backslash$ AS.met

Data: C: $\backslash$ EZStart $\backslash$ Projects $\backslash$ Default $\backslash$ Data $\backslash I-T M-35$

User: System

Acquired: $\quad$ 11/6/2006 5:24:32 PM

Printed: $\quad 12 / 13 / 2006$ 10:35:19 AM

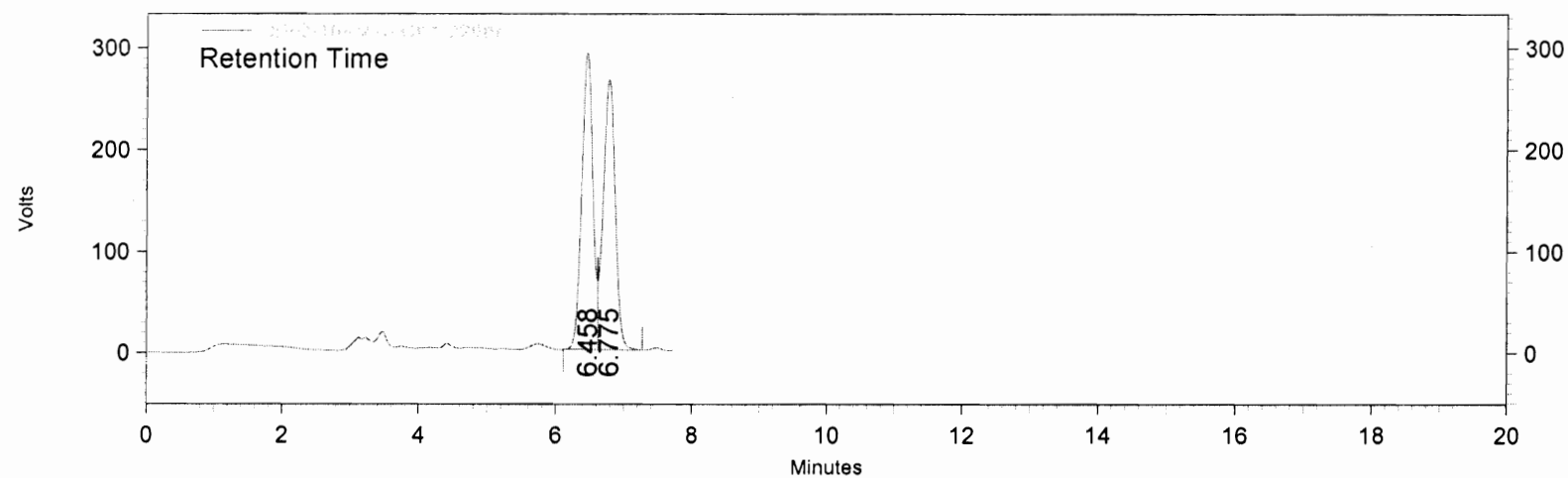

SPD-10AVvp

Ch1-220nm

Results

Retention Time

Area

Area \%

Height

Height \%

6.458

3305944

50.15

292435

52.32

6.775

3286216

49.85

266503

47.68

\begin{tabular}{|r|r|r|r|r|}
\hline Totals & 6592160 & 100.00 & 558938 & 100.00 \\
\hline
\end{tabular}<smiles>COCP(=O)(CC(O)(C[N+](=O)[O-])c1ccccc1)OC</smiles>

(5b, Racemic) 


\section{Area \% Report}

Method Name: C: $\backslash$ EZStart $\backslash$ Projects $\backslash$ Default $\backslash$ Methods $\backslash$ AS.met

Data: $\quad$ C: $\backslash$ EZStart $\backslash$ Projects $\backslash$ Default|Data $\backslash I-T M-123$

User: System

Acquired: $\quad$ 12/8/2006 10:15:15 AM

Printed: $\quad 12 / 13 / 2006$ 10:32:14 AM

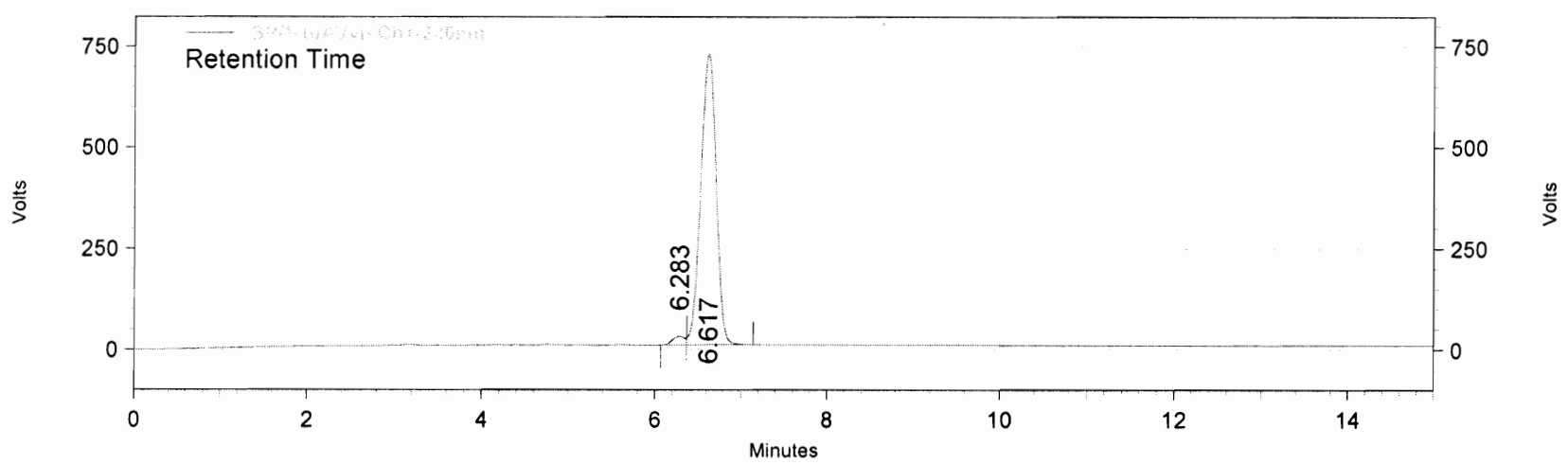

SPD-10AVvp

Ch1-220nm

Results

Retention Time

6.283

Area

Area \%

Height

Height \%

6.617

8961844

97.68

719754

97.11

\begin{tabular}{|r|r|r|r|r|}
\hline Totals & 9174308 & 100.00 & 741198 & 100.00 \\
\hline
\end{tabular}<smiles>COP(=O)(OC)C(O)(C[N+](=O)[O-])c1ccccc1</smiles>

(5b, $95 \%$ ee)

catalyst 3 


\section{Area \% Report}

Method Name: C: $\backslash$ EZStart $\backslash$ Projects $\backslash$ Default $\backslash$ Methods $\backslash A S$.met

Data: C: $\quad$ EZStart $\backslash$ Projects $\backslash$ Default $\backslash$ Data $\backslash I-T M-680 J$

User: System

Acquired: $\quad$ 11/19/2006 1:09:00 PM

Printed: $\quad$ 12/13/2006 10:36:03 AM

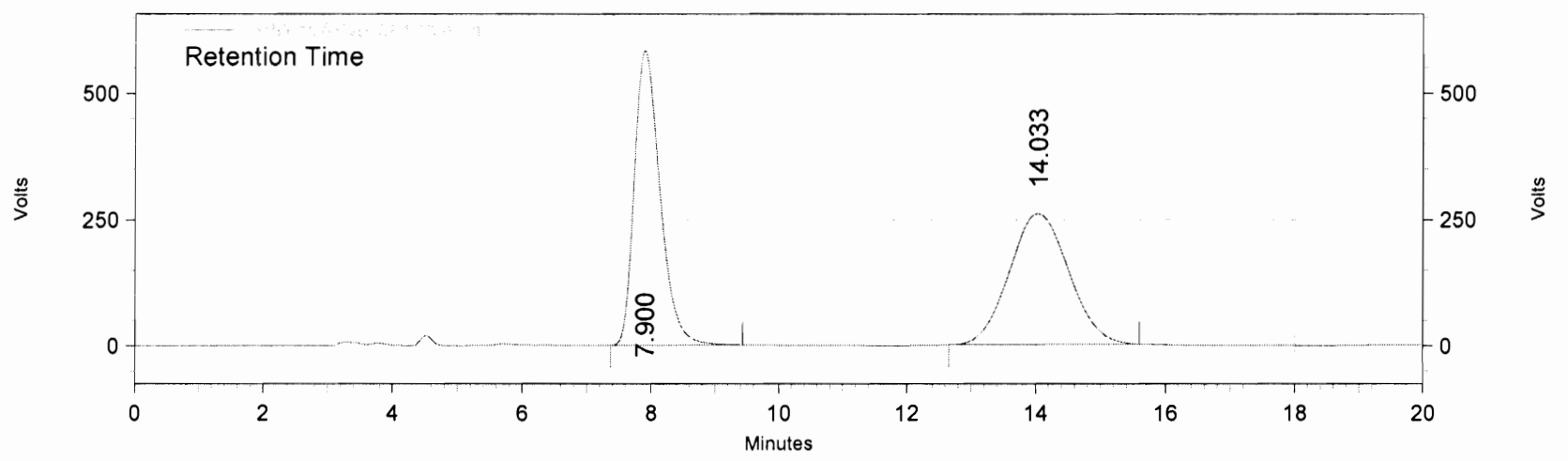

SPD-10AVvp

Ch1-220nm

Results

Retention Time

7.900

Area

Area \%

Height

Height \%

14.033

16276453

49.59

583350

69.25

16545745

50.41

259003

30.75

Totals<smiles>O=[N+]([O-])CC(O)(c1ccccc1)P(=O)(O[Ga])O[Ga]</smiles>

(5c, Racemic) 


\section{Area \% Report}

Method Name: C: $\mid$ EZStart $\mid$ Projects $\backslash$ Default $\mid$ Methods $\backslash A S$.met

Data:

User:

C:\EZStart|Projects\Default $\backslash$ Data $\backslash$ I-TM-30OJ

System

Acquired: $\quad$ 11/19/2006 1:42:07 PM

Printed:

12/13/2006 10:36:29 AM

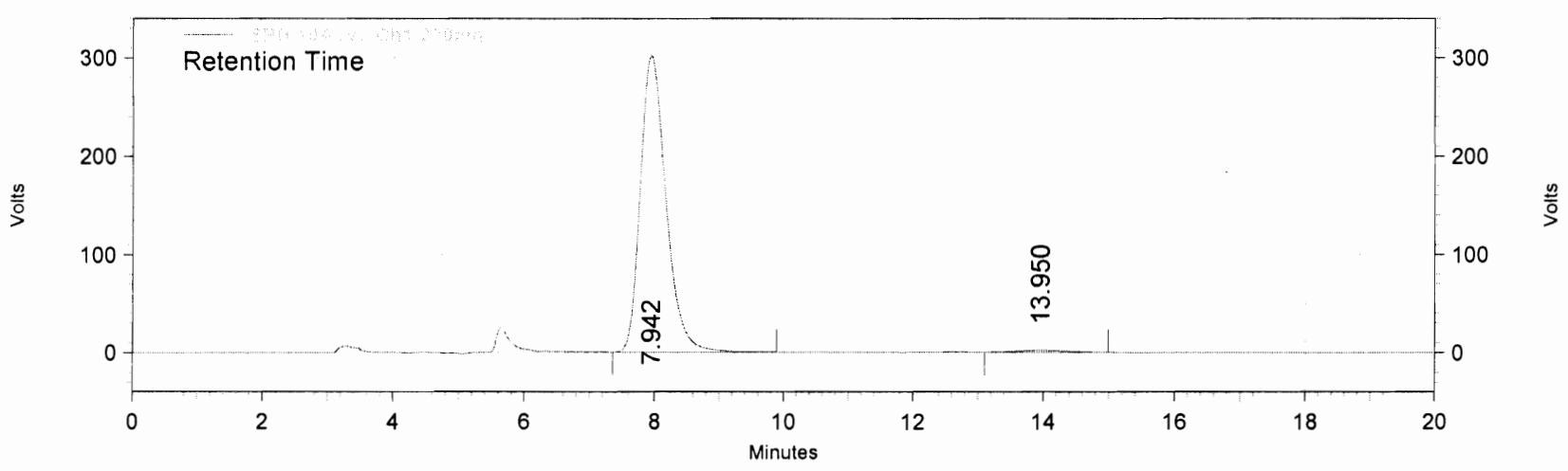

SPD-10AVvp

Ch1-220nm

Results

Retention Time

7.942

Area

Area \%

Height

98.75

301904

1893

Height \%

13.950

104827

1.25

0.62

Totals<smiles>CCCOP(=O)(OC(C)C)C(O)(C[N+](=O)[O-])c1ccccc1</smiles>

(5c, $98 \%$ ee)

catalyst 2 


\section{Area \% Report}

Method Name: C: $\backslash$ EZStart $\backslash$ Projects $\backslash$ Default $\backslash$ Methods $\backslash$ AS. met

Data: $\quad C: \backslash$ EZStart $\backslash$ Projects $\backslash$ Default $\backslash$ Data $\backslash I-T M-39 G$

User: $\quad$ System

Acquired: $\quad 11 / 17 / 2006$ 3:55:48 PM

Printed: $\quad 12 / 13 / 2006$ 10:39:48 AM

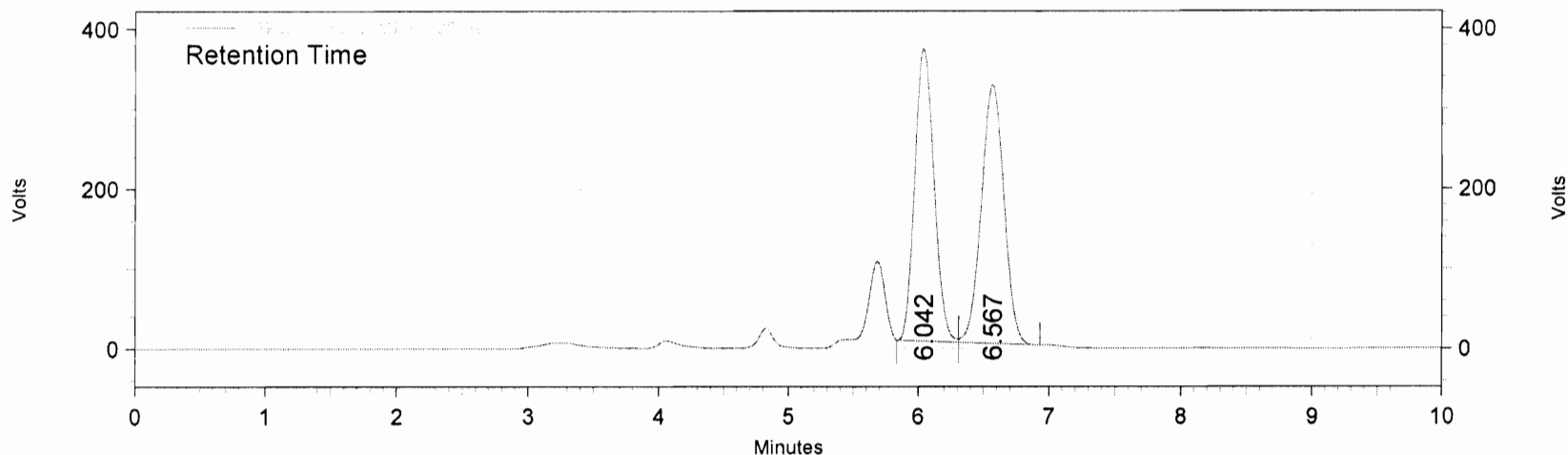

SPD-10AVvp

Ch1-220nm

Results

Retention Time

Area

Area \% Height

Height \%

6.042

3770875

49.18

366197

53.05

6.567

3896317

50.82

324138

46.95

\begin{tabular}{|r|r|r|r|r|}
\hline Totals & 7667192 & 100.00 & 690335 & 100.00 \\
\hline
\end{tabular}<smiles>COP(=O)(OC)C(O)(C[N+](=O)[O-])c1ccc(F)cc1</smiles>

(5d, Racemic) 


\section{Area \% Report}

Method Name: C: $\backslash$ EZStart $\backslash$ Projects $\backslash$ Default $\backslash$ Methods $\backslash$ AS.met

Data: C: $\quad$ EZStart $\backslash$ Projects $\backslash$ Default $\backslash$ Data $\backslash I-T M-42 P$

User: System

Acquired: $\quad$ 11/17/2006 4:45:24 PM

Printed: $\quad$ 12/13/2006 2:47:27 PM

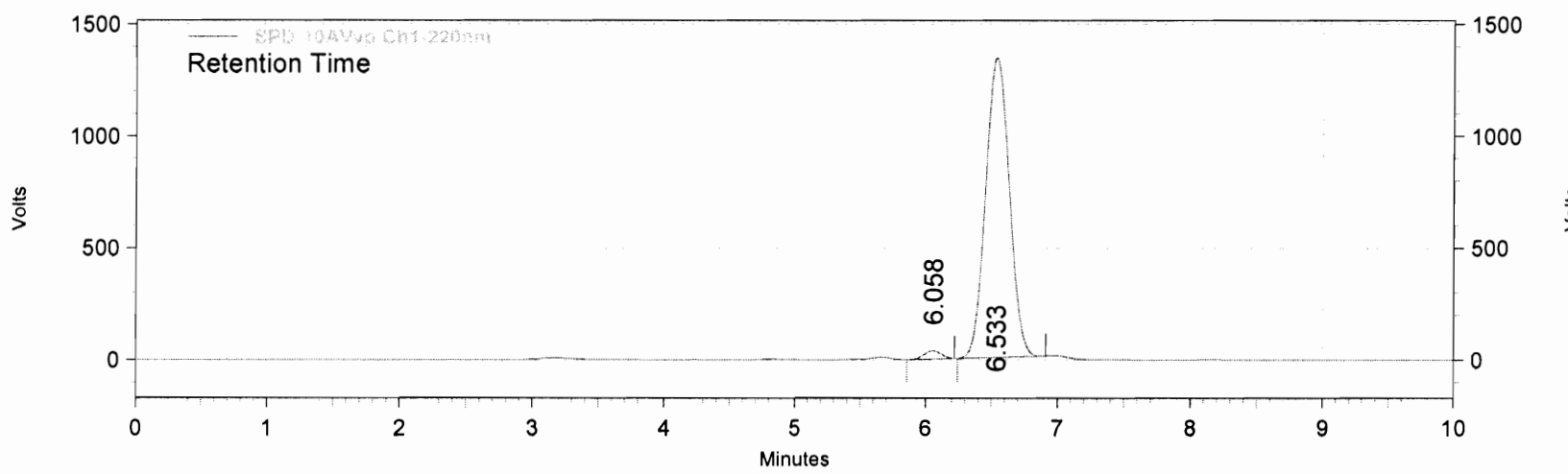

\section{SPD-10AVvp}

\section{Ch1-220nm}

Results

Retention Time

6.058

Area

Area \%

Height

Height \%

6.533

16724650

98.10

35896

1339245

97.39

\begin{tabular}{|r|r|r|r|r|}
\hline Totals & 17048708 & 100.00 & 1375141 & 100.00 \\
\hline
\end{tabular}<smiles>COP(=O)(OC)[C@](O)(C[N+](=O)[O-])c1ccc(F)cc1</smiles>

(5d, $96 \%$ ee)

catalyst 2 


\section{Area \% Report}

Method Name: C: $\backslash$ EZStart $\backslash$ Projects $\backslash$ Default $\backslash$ Methods $\backslash$ AS.met

Data: $\quad C: \backslash$ EZStart $\backslash$ Projects $\backslash$ Default $\backslash$ Data $\backslash I-T M-20 A D$

User: $\quad$ System

Acquired: $\quad$ 11/22/2006 3:47:20 PM

Printed: $\quad 12 / 13 / 2006$ 10:41:15 AM

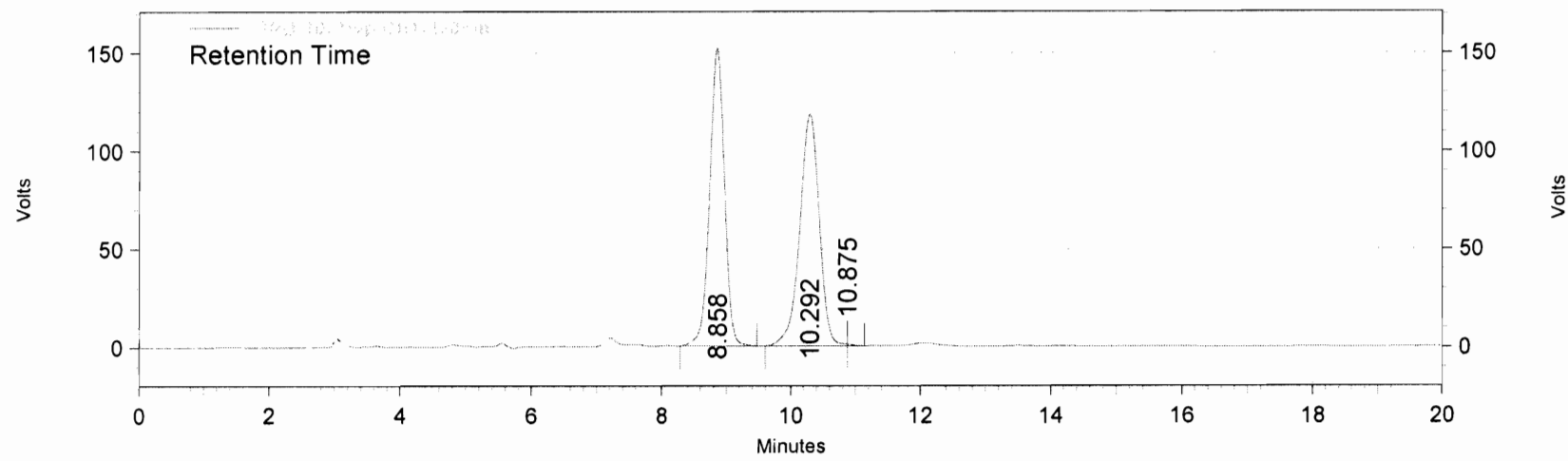

SPD-10AVvp

Ch1-220nm

Results

Retention Time

8.858

Area

Area \%

49.31

Height

151600

Height \%

10.292

2475061

50.59

118364

55.98

10.875

5081

0.10

846

Totals<smiles>CCOP(=O)(OCC)C(O)(C[N+](=O)[O-])c1ccc(F)cc1</smiles>

(5e, Racemic) 


\section{Area \% Report}

Method Name: C: $\backslash$ EZStart $\backslash$ Projects $\backslash$ Default $\backslash$ Methods $\backslash$ AS.met

Data: $\quad$ C: $\backslash$ EZStart $\backslash$ Projects $\backslash$ Default $\backslash$ Data $\backslash I-T M-77 A D$

User: $\quad$ System

Acquired: $\quad$ 11/22/2006 4:46:40 PM

Printed: $\quad 12 / 13 / 2006$ 10:43:50 AM

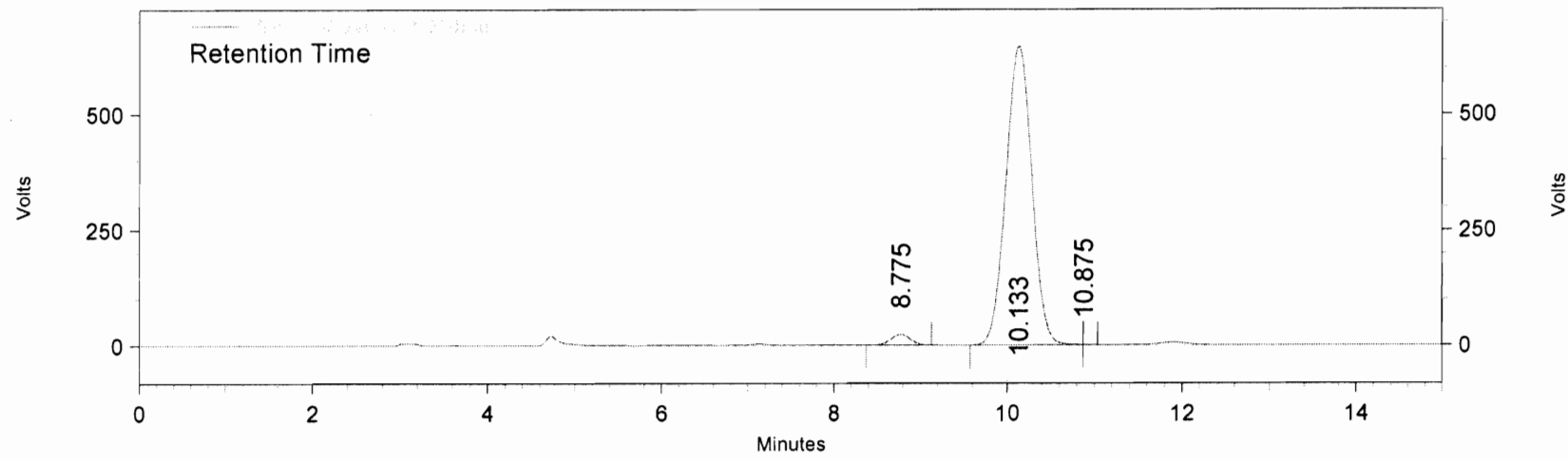

SPD-10AVvp

Ch1-220nm

Results

Retention Time

8.775

Area

Area $\%$

Height

22741

Height $\%$

10.133

13370025

3.40

10.875

1877

0.01

464

0.07

Totals<smiles>CCOP(=O)(OCC)[C@@](O)(C[N+](=O)[O-])c1ccc(F)cc1</smiles>

(5e, $95 \%$ ee) catalyst 2 


\section{Area \% Report}

Method Name: C: $\backslash$ EZStart $\backslash$ Projects $\backslash$ Default $\backslash$ Methods $\backslash$ AS.met

Data: C: $\quad$ EZStart $\backslash$ Projects $\backslash$ Default $\backslash$ Data $\backslash I-T M-59$

User: System

Acquired: $\quad$ 11/17/2006 12:59:20 PM

Printed: $\quad 12 / 13 / 2006$ 10:44:20 AM

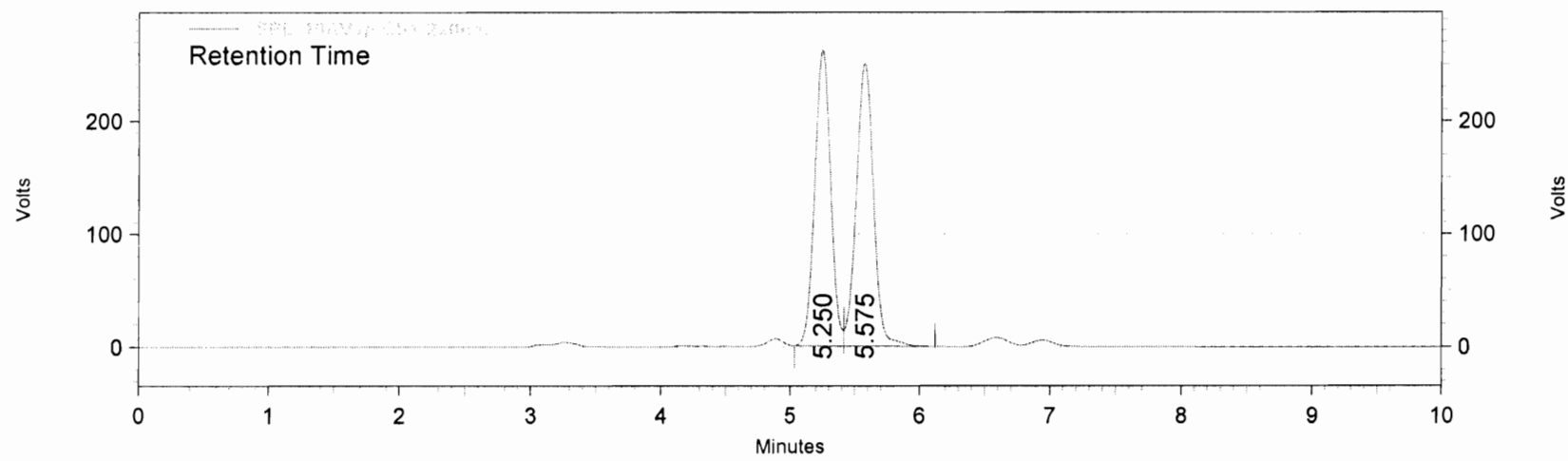

SPD-10AVvp

Ch1-220nm

Results

Retention Time

5.250

Area

Area $\%$ Height Height \%

5.575

49.19

262457

51.14

2306218

50.81

250720

48.86

\begin{tabular}{|r|r|r|r|r|}
\hline Totals & 4538949 & 100.00 & 513177 & 100.00 \\
\hline
\end{tabular}<smiles>CCCOP(=O)(OCC)C(O)(C[N+](=O)[O-])c1ccc(F)cc1</smiles>

(5f, Racemic) 


\section{Area \% Report}

Method Name: C:IEZStart $\mid$ Projects $\backslash$ Default $\backslash$ Methods $\backslash A S$. met

Data: C: $\quad$ EZStart|Projects $\backslash$ Default $\backslash$ Data $\backslash I-T M-43$

User: $\quad$ System

Acquired: $\quad$ 11/17/2006 2:00:21 PM

Printed: $\quad$ 12/13/2006 10:44:45 AM

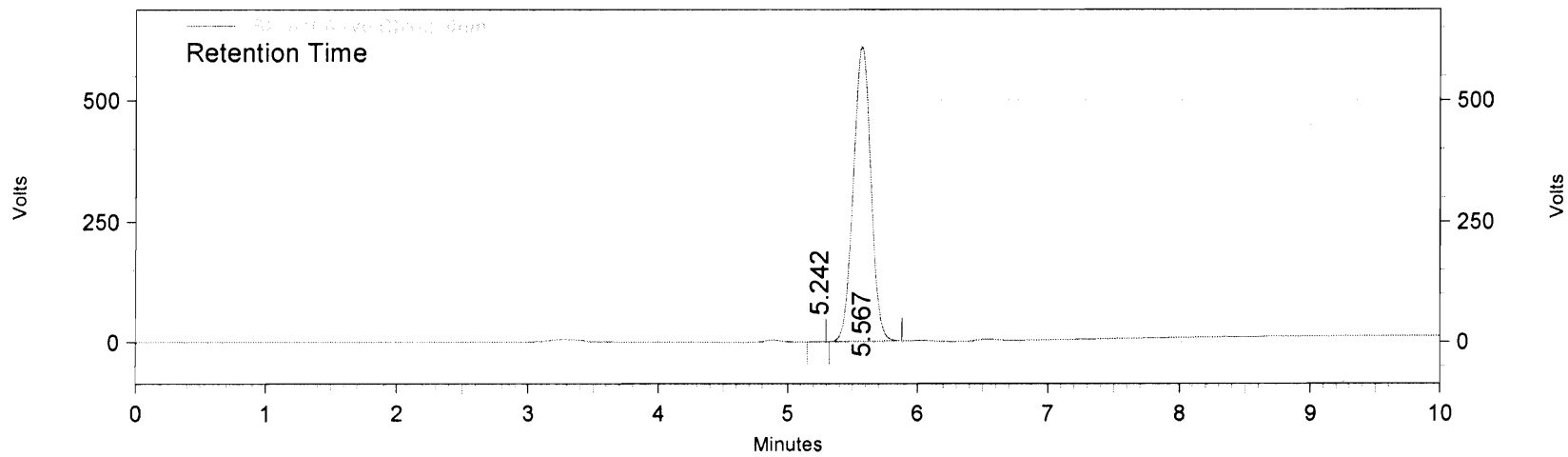

SPD-10AVvp

Ch1-220nm

Results

Retention Time

Area

Area \% Height

Height \%

5.242

2324

0.04

5721612

99.96

609449

99.92

\section{Totals}<smiles>CCCOP(=O)(OCC)C(O)(C[N+](=O)[O-])c1ccc(F)cc1</smiles>

(5f, $>99 \%$ ee)

catalyst 2 


\section{Area \% Report}

Method Name: C: $\backslash$ EZStart $\backslash$ Projects $\backslash$ Default $\backslash$ Methods $\backslash A S$.met

Data: C: $\backslash$ EZStart $\backslash$ Projects $\backslash$ Default $\backslash$ Data $\backslash$ I-TM-63OJ

User: System

Acquired: $\quad$ 11/18/2006 3:00:11 PM

Printed: 12/13/2006 10:45:11 AM

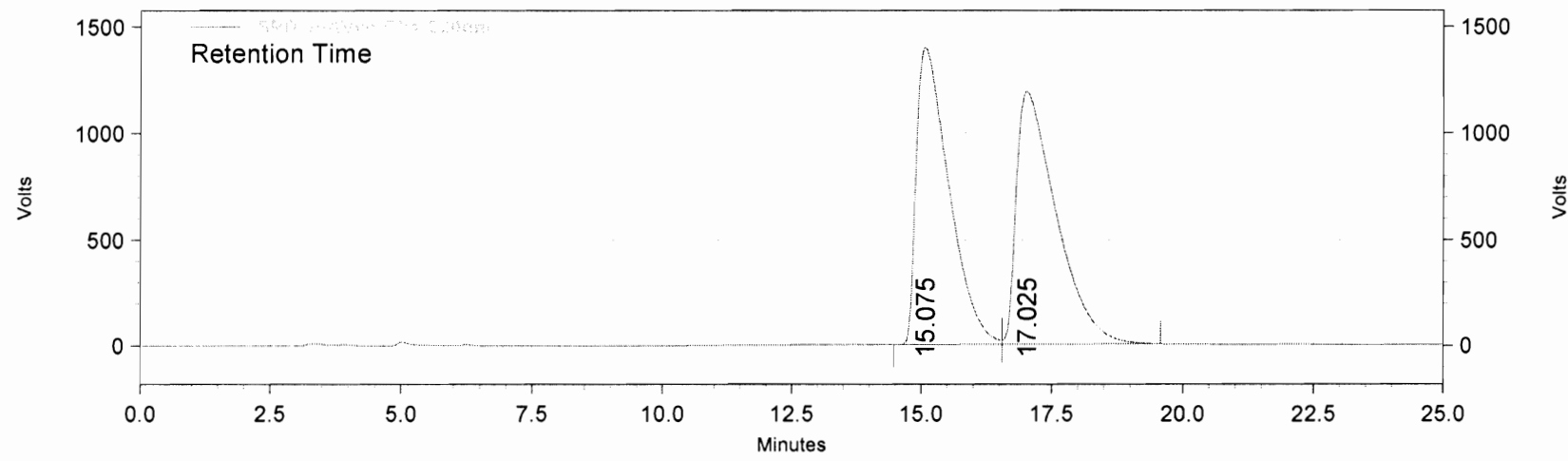

SPD-10AVvp

Ch1-220nm

Results

Retention Time

15.075

Area

Area \%

Height

Height $\%$

17.025

61200500

49.25

1396728

54.04

63067976

1187964

45.96

\begin{tabular}{|r|r|r|r|r|}
\hline Totals & 124268476 & 100.00 & 2584692 & 100.00 \\
\hline
\end{tabular}<smiles>CCOP(=O)(OCC)C(O)(C[N+](=O)[O-])c1cccc(F)c1</smiles>

(5g, Racemic) 


\section{Area \% Report}

Method Name: C:LEZStart $\mid$ Projects $\backslash$ Default $\backslash$ Methods $\backslash A S$.met

Data: $\quad$ C: $:$ EZStart $\backslash$ Projects $\backslash$ Default $\backslash$ DatalI-TM-640J

User: $\quad$ System

Acquired: $\quad$ 11/18/2006 3:36:58 PM

Printed: $\quad$ 12/13/2006 10:45:30 AM

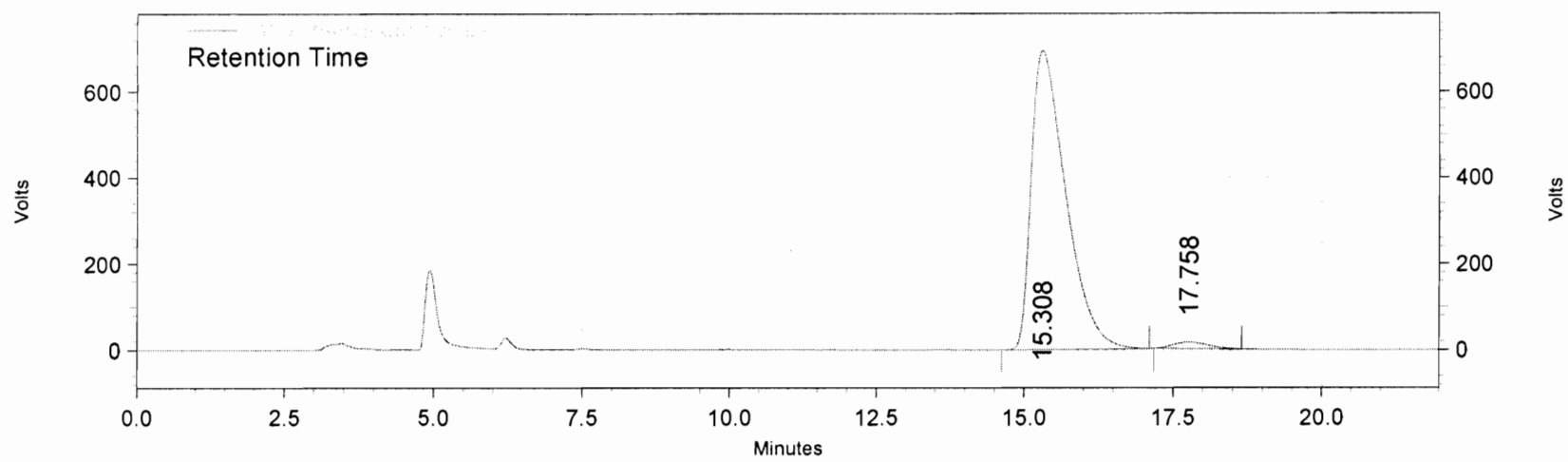

SPD-10AVvp

Ch1-220nm

Results

Retention Time

15.308

Area

Area \%

Height

Height \%

17.758

592342

2.08

14536

2.05

\begin{tabular}{|r|r|r|r|r|}
\hline Totals & 28528037 & 100.00 & 708922 & 100.00 \\
\hline
\end{tabular}<smiles>CCOP(=O)(OCC)C(O)(C[N+](=O)[O-])c1cccc(F)c1</smiles>

(5g, $96 \%$ ee)

catalyst 2 


\section{Area \% Report}

Method Name: C: $\backslash$ EZStart $\backslash$ Projects $\backslash$ Default $\backslash$ Methods $\backslash$ AS.met

Data:

User:

C: $\backslash$ EZStart $\backslash$ Projects $\backslash$ Default $\backslash$ Data $\backslash I-T M-27 A D$

11/4/2006 3:32:18 PM

Printed:

12/13/2006 10:36:54 AM

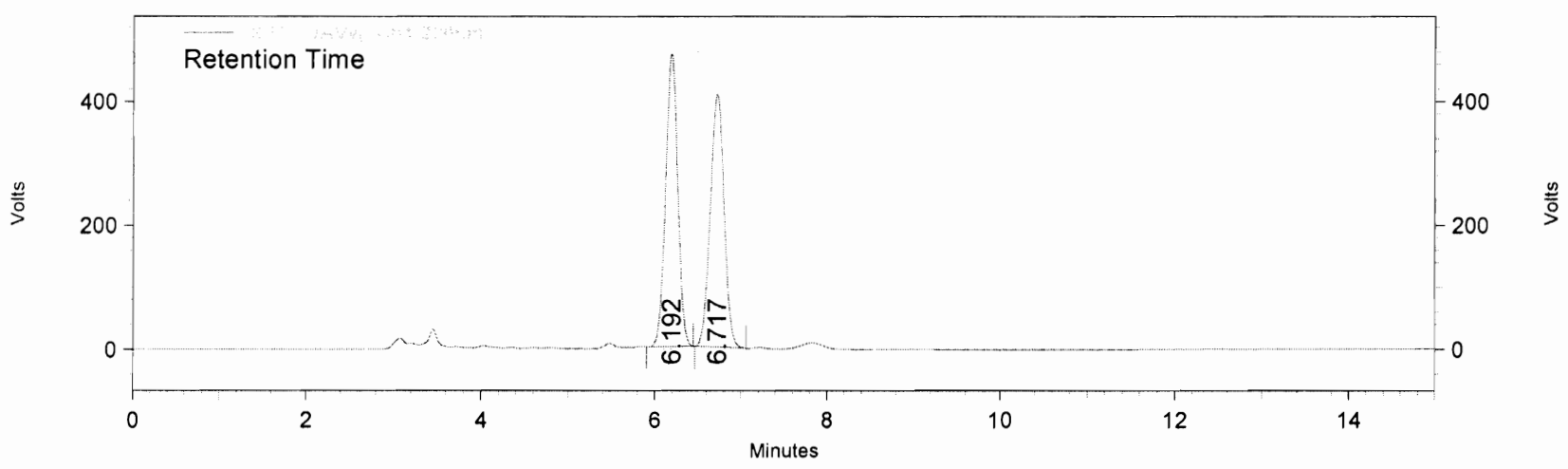

SPD-10AVvp

Ch1-220nm

Results

Retention Time

6.192

Area

Area \%

Height

473122

Height \%

6.717

4552166

49.53

409025

46.37

Totals<smiles>COP(=O)(OC)C(O)(C[N+](=O)[O-])c1ccc(Cl)cc1</smiles>

(5h, Racemic) 


\section{Area \% Report}

Method Name: C: $\backslash$ EZStart $\backslash$ Projects $\backslash$ Default $\backslash$ Methods $\backslash$ AS. met

Data:

User:

C: $\backslash$ EZStart $\backslash$ Projects $\backslash$ Default $\backslash$ Data $\backslash I-T M-58 A D 1$

System

Acquired: $\quad$ 11/17/2006 12:06:31 PM

Printed:

12/13/2006 10:37:42 AM

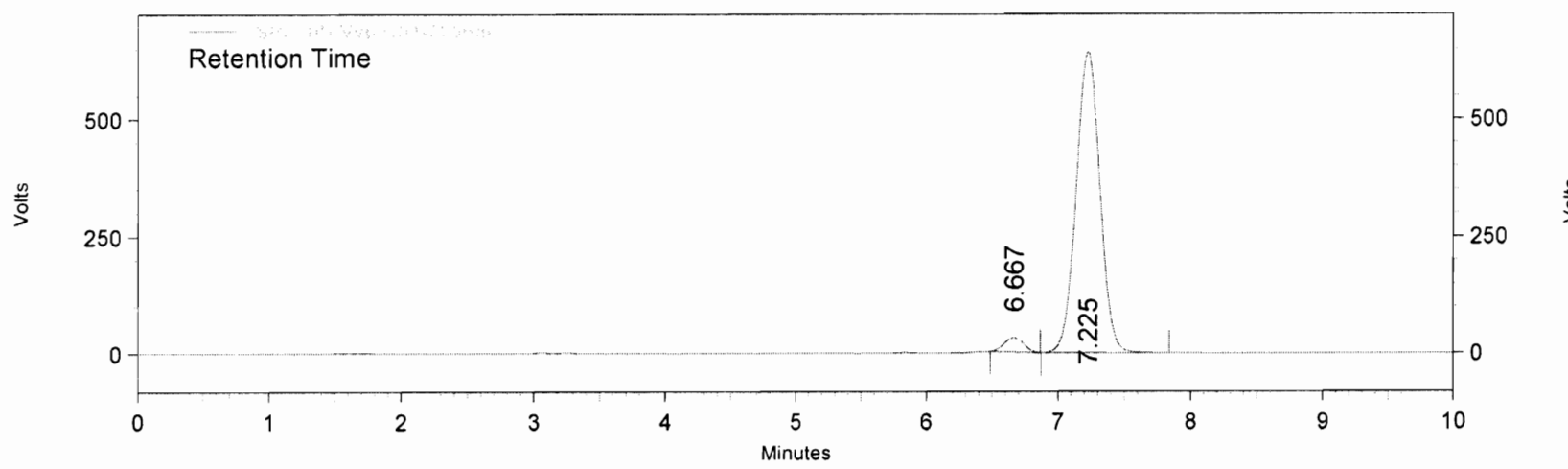

SPD-10AVvp

Ch1-220nm

Results

Retention Time

6.667

Area

Area \%

Height

30407

Height \%

7.225

7998275

96.32

643608

95.49

\begin{tabular}{|r|r|r|r|r|}
\hline Totals & 8304087 & 100.00 & 674015 & 100.00 \\
\hline
\end{tabular}<smiles>COP(=O)(OC)C(O)(C[N+](=O)[O-])c1ccc(Cl)cc1</smiles>

(5h, $93 \%$ ee)

catalyst 2 


\section{Area \% Report}

Method Name: C:IEZStart $\backslash$ Projects $\backslash$ Default $\backslash$ Methods $\backslash A S$.met

Data: C:IEZStart|Projects|Default|DatalI-TM-22

User: $\quad$ System

Acquired: $\quad$ 11/4/2006 10:06:02 AM

Printed: $\quad$ 12/13/2006 10:38:08 AM

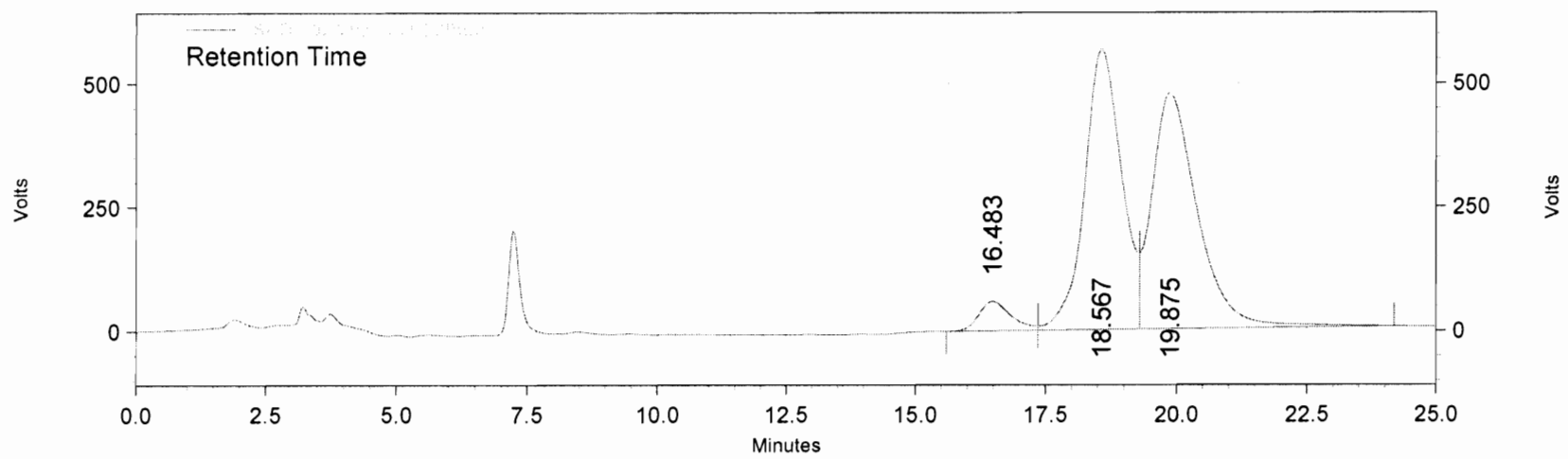

SPD-10AVvp

Ch1-220nm

Results

Retention Time

16.483

Area

Area \%

Height

Height \%

18.567

2557881

27817477

59091

$47.05 \quad 566013$

51.39

19.875

28743548

48.62

476237

43.24

Totals<smiles>CCOP(=O)(OCC)C(O)(C[N+](=O)[O-])c1ccc(Cl)cc1</smiles>

(5i, Racemic) 


\section{Area \% Report}

Method Name: C:|EZStart|Projects\Default $\backslash$ Methods $\backslash A S$.met

Data: C: $\quad$ EZStart|Projects $\backslash$ Default|Data|I-TM-24

User: $\quad$ System

Acquired: $\quad$ 11/4/2006 10:47:14 AM

Printed: $\quad$ 12/13/2006 10:38:34 AM

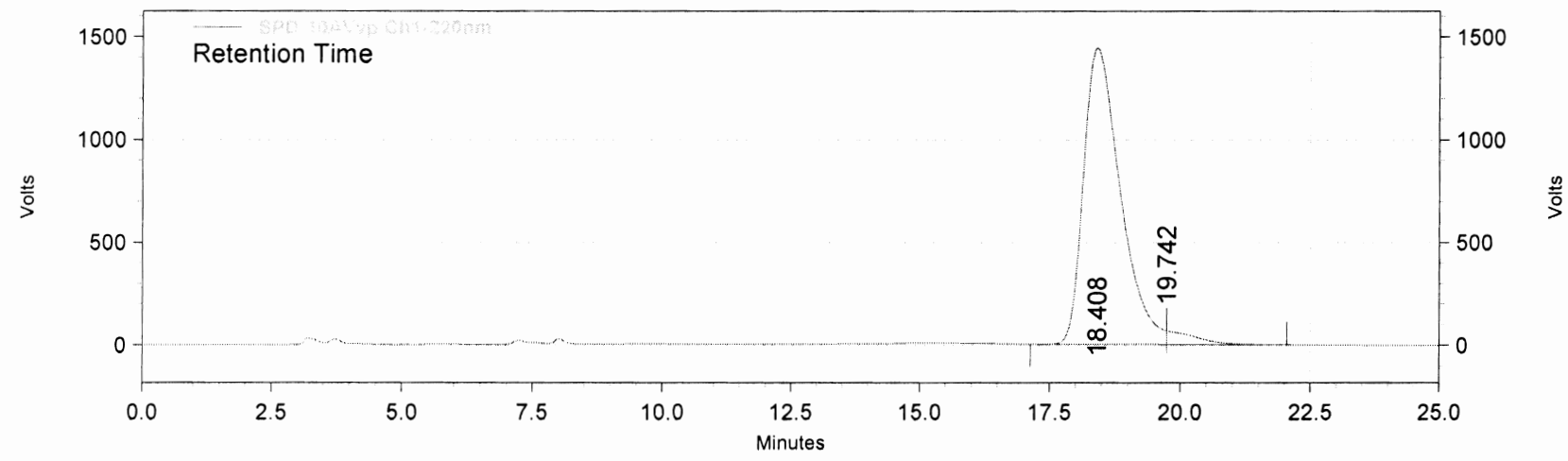

SPD-10AVvp

Ch1-220nm

Results

Retention Time

Area

Area \%

Height

Height \%

19.742

2588082

3.53

441821

95.63

Totals<smiles>CCOP(=O)(OCC)C(O)(C[N+](=O)[O-])c1ccc(Cl)cc1</smiles>

(5i, $93 \%$ ee)

catalyst 2 


\section{Area \% Report}

Method Name: C: $\backslash$ EZStart $\backslash$ Projects $\backslash$ Default $\backslash$ Methods $\backslash$ AS.met

Data: $\quad C: \backslash E Z S t a r t \backslash$ Projects $\backslash$ Default $\backslash$ Data $\backslash I-T M-37$

User: $\quad$ System

Acquired: $\quad 11 / 7 / 2006$ 11:37:35 AM

Printed: $\quad 12 / 13 / 2006$ 10:38:56 AM

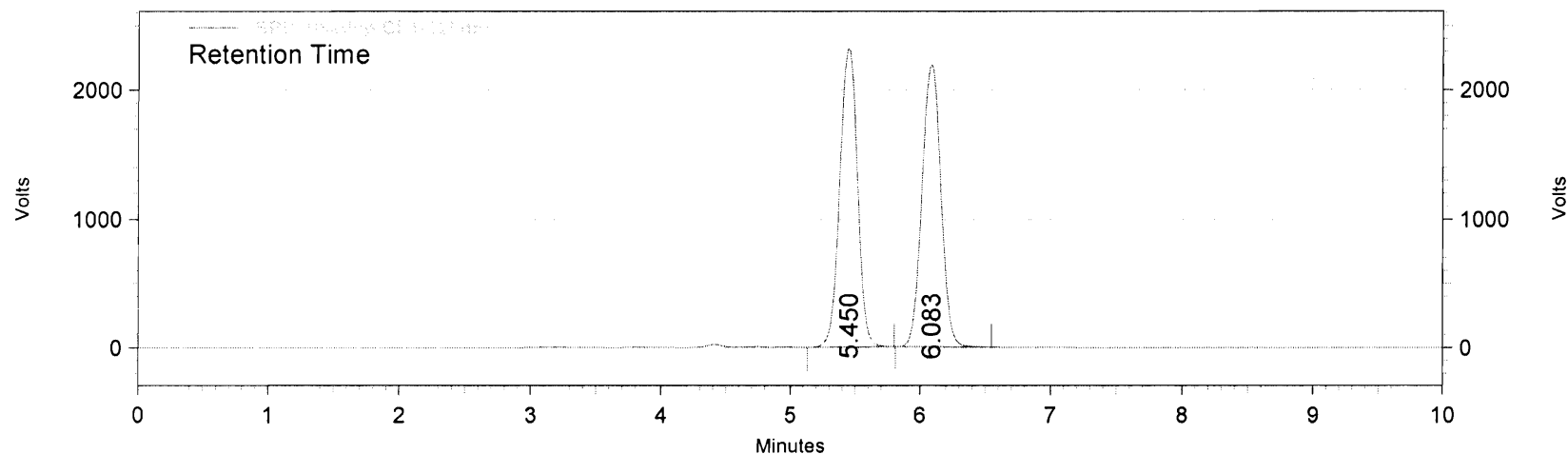

SPD-10AVvp

Ch1-220nm

Results

Retention Time

Area

Area \%

Height

Height \%

5.450

22086450

49.84

2318980

51.45

6.083

22227085

50.16

2188501

48.55

\begin{tabular}{|r|r|r|r|r|}
\hline Totals & 44313535 & 100.00 & 4507481 & 100.00 \\
\hline
\end{tabular}<smiles>CCCOP(=O)(OC(C)C)C(O)(C[N+](=O)[O-])c1ccc(Cl)cc1</smiles>

(5j, Racemic) 


\section{Area \% Report}

Method Name: C: $\backslash$ EZStart $\backslash$ Projects $\backslash$ Default $\backslash$ Methods $\backslash$ AS. met

Data: C: $\quad$ EZStart $\backslash$ Projects $\backslash$ Default $\backslash$ Data $\backslash I-T M-33 P 1$

User: System

Acquired: $\quad 11 / 17 / 2006$ 12:32:07 PM

Printed: $\quad 12 / 13 / 2006$ 10:39:20 AM

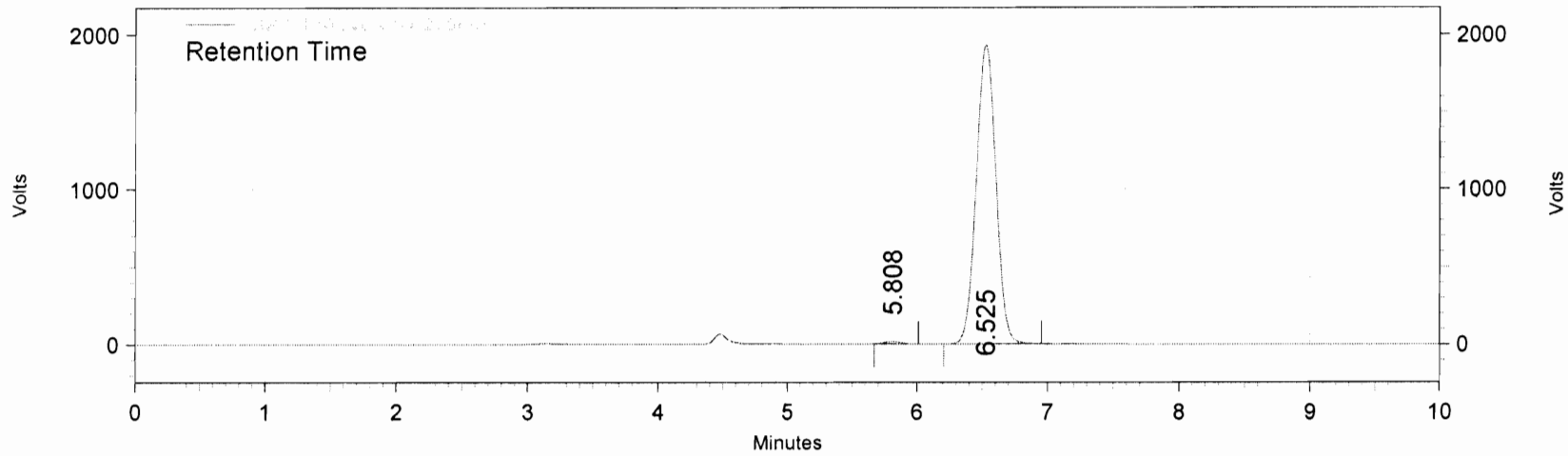

SPD-10AVvp

Ch1-220nm

Results

Retention Time

Area

Area \%

Height

Height \%

5.808

130995

0.64

15469

0.79

6.525

20193282

99.36

1931561

99.21

\begin{tabular}{|r|r|r|r|r|}
\hline Totals & 20324277 & 100.00 & 1947030 & 100.00 \\
\hline
\end{tabular}<smiles>CCCOP(=O)(OC(C)C)C(O)(C[N+](=O)[O-])c1ccc(Cl)cc1</smiles>

(5j, 99\% ee)

catalyst 2 


\section{Area \% Report}

Method Name: C: $\backslash$ EZStart $\backslash$ Projects $\backslash$ Default $\backslash$ Methods $\backslash A S$.met

Data: $\quad$ C: $\backslash$ EZStart $\backslash$ Projects $\backslash$ Default $\backslash$ Data $\backslash$ I-TM-720J

User: $\quad$ System

Acquired: $\quad 11 / 21 / 200611: 39: 54 \mathrm{AM}$

Printed: $\quad$ 12/13/2006 2:26:45 PM

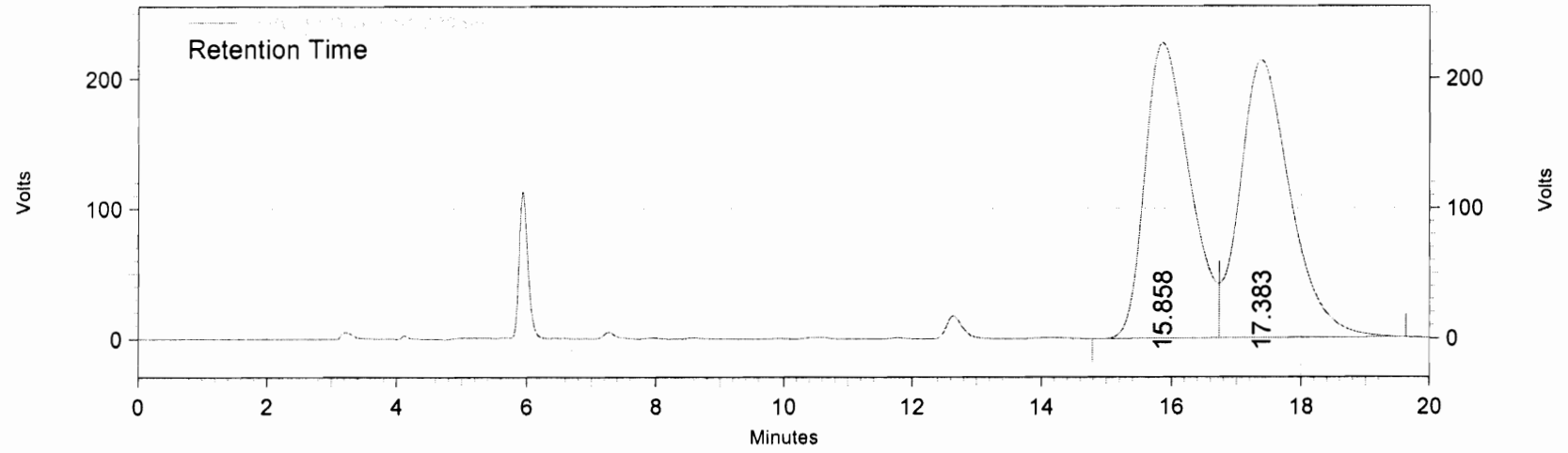

SPD-10AVvp

Ch1-220nm

Results

Retention Time

15.858

Area

Area \%

48.34

Height

Height \%

17.383

11547502

51.66

213490

48.45

\begin{tabular}{|r|r|r|r|r|}
\hline Totals & 22351990 & 100.00 & 440635 & 100.00 \\
\hline
\end{tabular}<smiles>CCOP(=O)(OCC)C(O)(C[N+](=O)[O-])c1ccc(Br)cc1</smiles>

(5k, Racemic) 


\section{Area \% Report}

Method Name: C: $\backslash$ EZStart $\backslash$ Projects $\backslash$ Default $\backslash$ Methods $\backslash A S$.met

Data: C: $\backslash$ EZStart $\backslash$ Projects $\backslash$ Default $\backslash$ Data $\backslash I-T M-730 J$

User: $\quad$ System

Acquired: $\quad 11 / 21 / 200611: 15: 37 \mathrm{AM}$

Printed: $\quad 12 / 13 / 2006$ 2:27:06 PM

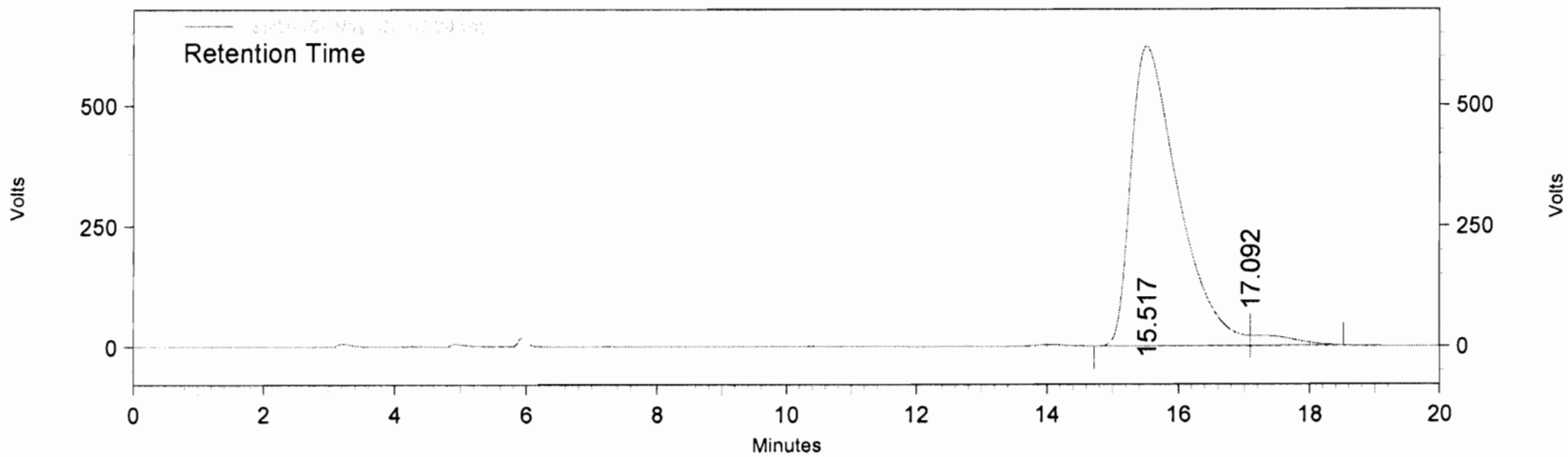

SPD-10AVvp

Ch1-220nm

Results

Retention Time

15.517

Area

Area \%

Height

621107

Height \%

17.092

927172

2.89

20329

3.17

\begin{tabular}{|r|r|r|r|r|}
\hline Totals & 32064369 & 100.00 & 641436 & 100.00 \\
\hline
\end{tabular}<smiles>CCOP(=O)(OCC)C(O)(C[N+](=O)[O-])c1ccc(Br)cc1</smiles>

(5k, $94 \%$ ee)

catalyst 2 


\section{Area \% Report}

Method Name: C: $\backslash$ EZStart $\backslash$ Projects $\backslash$ Default $\backslash$ Methods $\backslash A S$. met

Data: C: $\quad$ EZStart $\backslash$ Projects $\backslash$ Default $\backslash$ Data $\backslash I-T M-60$

User: $\quad$ System

Acquired: $\quad 11 / 17 / 2006$ 2:33:20 PM

Printed: $\quad$ 12/13/2006 2:26:09 PM

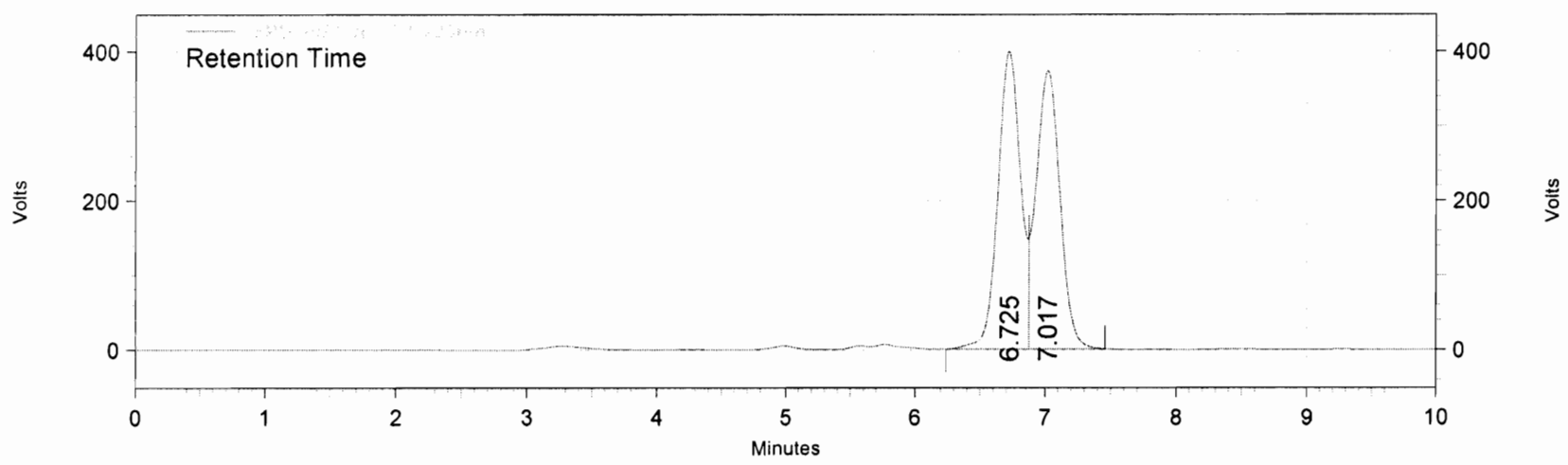

SPD-10AVvp

Ch1-220nm

Results

Retention Time

Area \%

Height

Height $\%$

6.725

4801859

50.42

398061

51.64

7.017

4722059

49.58

372733

48.36

\begin{tabular}{|r|r|r|r|r|}
\hline Totals & 9523918 & 100.00 & 770794 & 100.00 \\
\hline
\end{tabular}<smiles>COP(=O)(OC)C(O)(C[N+](=O)[O-])c1ccc(C)cc1</smiles>

(5I, Racemic) 


\section{Area \% Report}

Method Name: C: $\backslash$ EZStart $\backslash$ Projects $\backslash$ Default $\backslash$ Methods $\backslash$ AS.met

Data: C: $\quad$ EZStart $\backslash$ Projects $\backslash$ Default $\backslash$ Data $\backslash I-T M-129$

User: $\quad$ System

Acquired: $\quad$ 12/10/2006 12:56:52 PM

Printed: $\quad$ 12/13/2006 10:31:50 AM

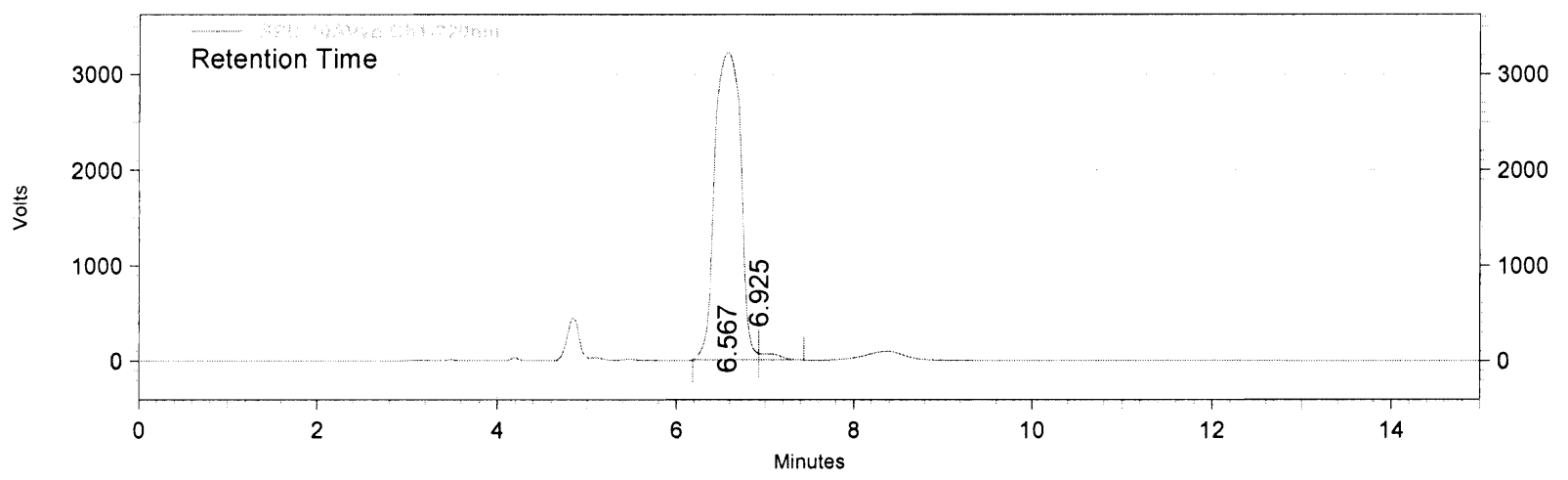

SPD-10AVvp

Ch1-220nm

Results

Retention Time

6.567

Area

Area \%

Height

Height \%

6.925

98.48

3213717

65581

2.00

\begin{tabular}{|r|r|r|r|r|}
\hline Totals & 66038802 & 100.00 & 3279298 & 100.00 \\
\hline
\end{tabular}<smiles>COP(=O)(OC)[C@](O)(C[N+](=O)[O-])c1ccc(C)cc1</smiles>

(5I, $97 \%$ ee)

catalyst 3 


\section{Area \% Report}

Method Name: C: $\mid$ EZStart $\mid$ Projects $\backslash$ Default $\backslash$ Methods $\backslash A S$.met

Data: C:IEZStart|Projects|Default|DatalI-TM-23

User: $\quad$ System

Acquired: $\quad 11 / 5 / 2006$ 11:42:08 AM

Printed: $\quad$ 12/13/2006 2:25:04 PM

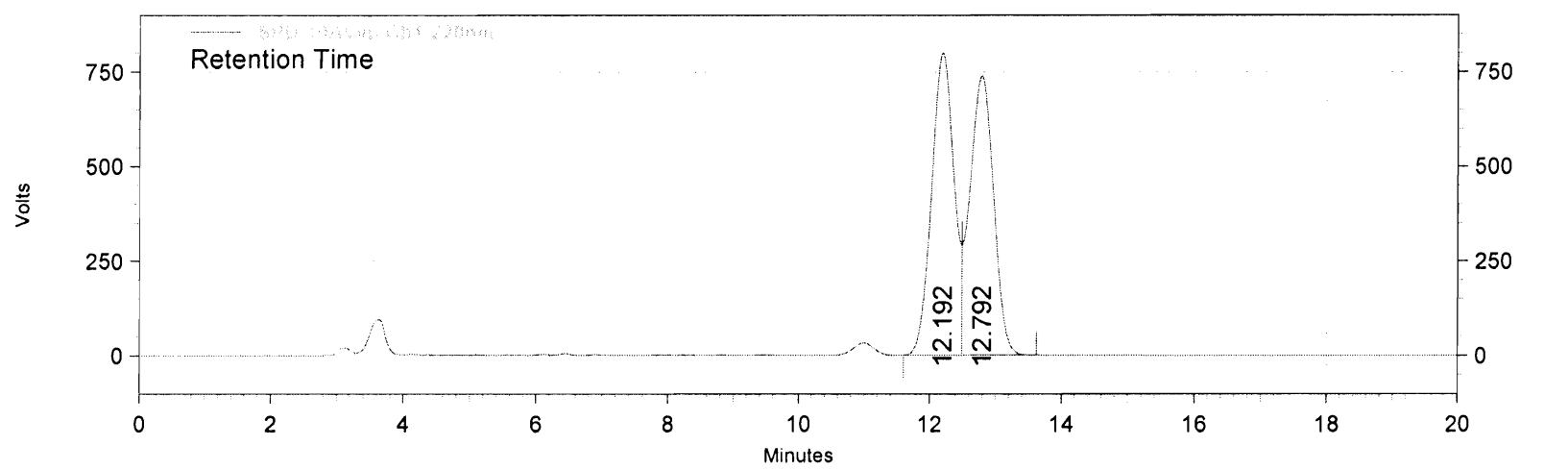

SPD-10AVvp

Ch1-220nm

Results

Retention Time

12.192

Area

Area \%

Height

Height \%

12.792

18538828

50.50

798230

51.97

49.50

48.03

\begin{tabular}{|r|r|r|r|r|}
\hline Totals & 37451162 & 100.00 & 1535951 & 100.00 \\
\hline
\end{tabular}<smiles>CCOP(=O)(OCC)C(O)(C[N+](=O)[O-])c1ccc(C)cc1</smiles>

(5m, Racemic) 


\section{Area \% Report}

Method Name: C: $\backslash$ EZStart $\backslash$ Projects $\backslash$ Default $\backslash$ Methods $\backslash$ AS.met

Data: C: CEZStart $\backslash$ Projects $\backslash$ Default $\backslash$ Data $\backslash I-T M-26$

User: System

Acquired: $\quad$ 11/5/2006 1:56:25 PM

Printed: $\quad$ 12/13/2006 2:24:48 PM

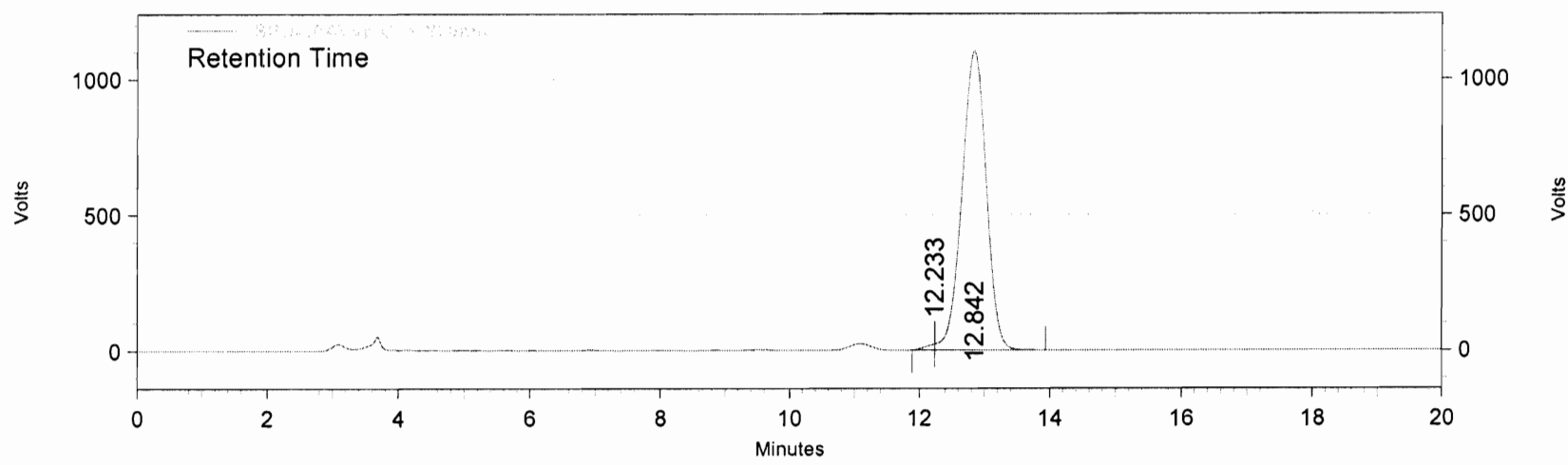

SPD-10AVvp

Ch1-220nm

Results

Retention Time

12.233

Area

Area \%

Height

Height \%

12.842

28483741

99.31

20783

1.85

\begin{tabular}{|r|r|r|r|r|}
\hline Totals & 28680878 & 100.00 & 1122188 & 100.00 \\
\hline
\end{tabular}<smiles>CCOP(=O)(OCC)C(O)(C[N+](=O)[O-])c1ccc(C)cc1</smiles>

(5m, $99 \%$ ee)

catalyst 2 


\section{Area \% Report}

Method Name: C: $\backslash$ EZStart $\backslash$ Projects $\backslash$ Default $\backslash$ Methods $\backslash$ AS.met

Data: C: $\backslash$ EZStart $\backslash$ Projects $\backslash$ Default $\backslash$ Data $\backslash$ I-TM-65OJ

User: $\quad$ System

Acquired: $\quad$ 11/21/2006 3:30:59 PM

Printed: $\quad$ 12/13/2006 2:24:24 PM

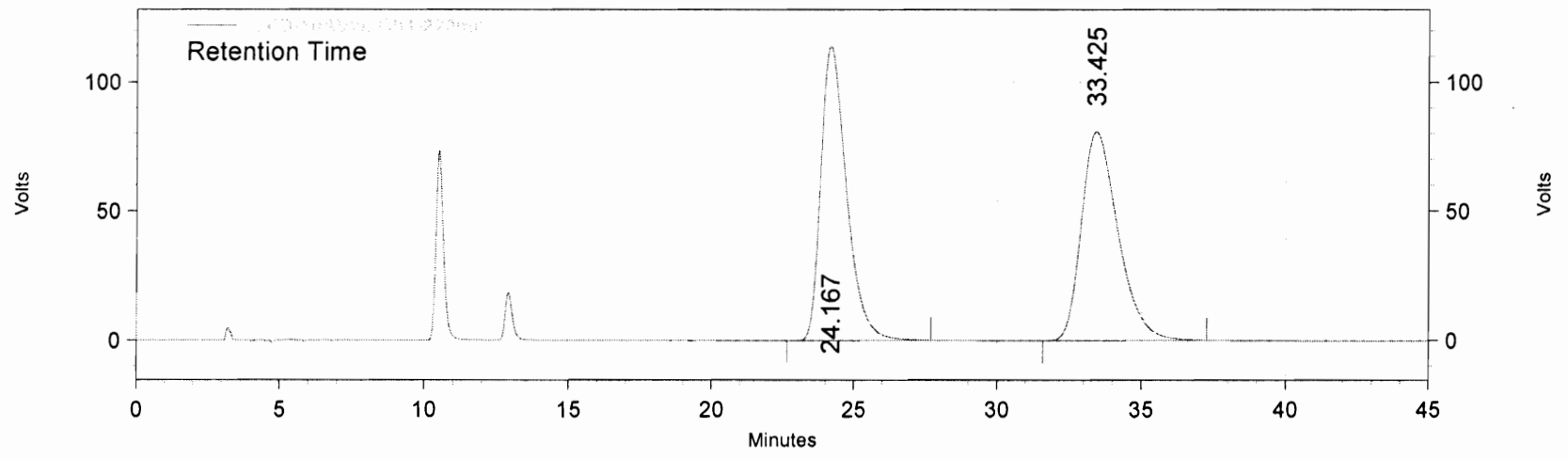

SPD-10AVvp

Ch1-220nm

Results

Retention Time

Area

Area $\%$

Height

Height $\%$

24.167

7130464

50.05

49.95

114103

58.55

33.425

7116127

80765

41.45

\begin{tabular}{|r|r|r|r|r|}
\hline Totals & 14246591 & 100.00 & 194868 & 100.00 \\
\hline
\end{tabular}<smiles>CCOP(=O)(OCC)C(O)(C[N+](=O)[O-])c1ccc(OC)cc1</smiles>

(5n, Racemic) 


\section{Area \% Report}

Method Name: C: $\backslash$ EZStart $\backslash$ Projects $\backslash$ Default $\backslash$ Methods $\backslash$ AS.met

Data: $\quad$ C: $\backslash E Z S t a r t \backslash P r o j e c t s \backslash D e f a u l t \backslash D a t a \backslash I-T M-67$

User: $\quad$ System

Acquired: $\quad 11 / 21 / 2006$ 2:38:53 PM

Printed: $\quad 12 / 13 / 2006$ 2:23:50 PM

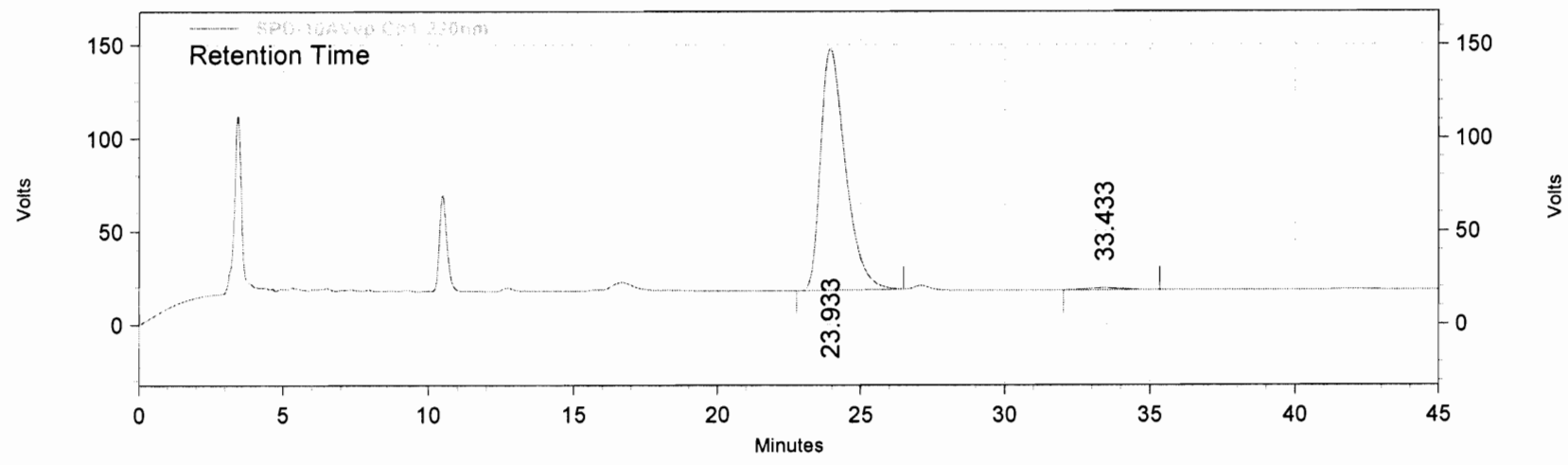

SPD-10AVvp

Ch1-220nm

Results

Retention Time

23.933

Area

Area \%

98.91

Height

Height \%

33.433

86266

1.09

1044

0.80

\begin{tabular}{|r|r|r|r|r|}
\hline Totals & 7926095 & 100.00 & 130794 & 100.00 \\
\hline
\end{tabular}<smiles>CCOP(=O)(OCC)C(O)(C[N+](=O)[O-])c1ccc(OC)cc1</smiles>

(5n, $98 \%$ ee)

catalyst 2 


\section{Area \% Report}

Method Name: C: $\backslash$ EZStart $\backslash$ Projects $\backslash$ Default $\backslash$ Methods $\backslash$ AS. met

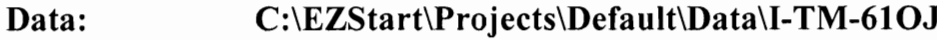

User: $\quad$ System

Acquired: $\quad$ 11/18/2006 10:08:36 AM

Printed: $\quad 12 / 13 / 2006$ 11:03:18 AM

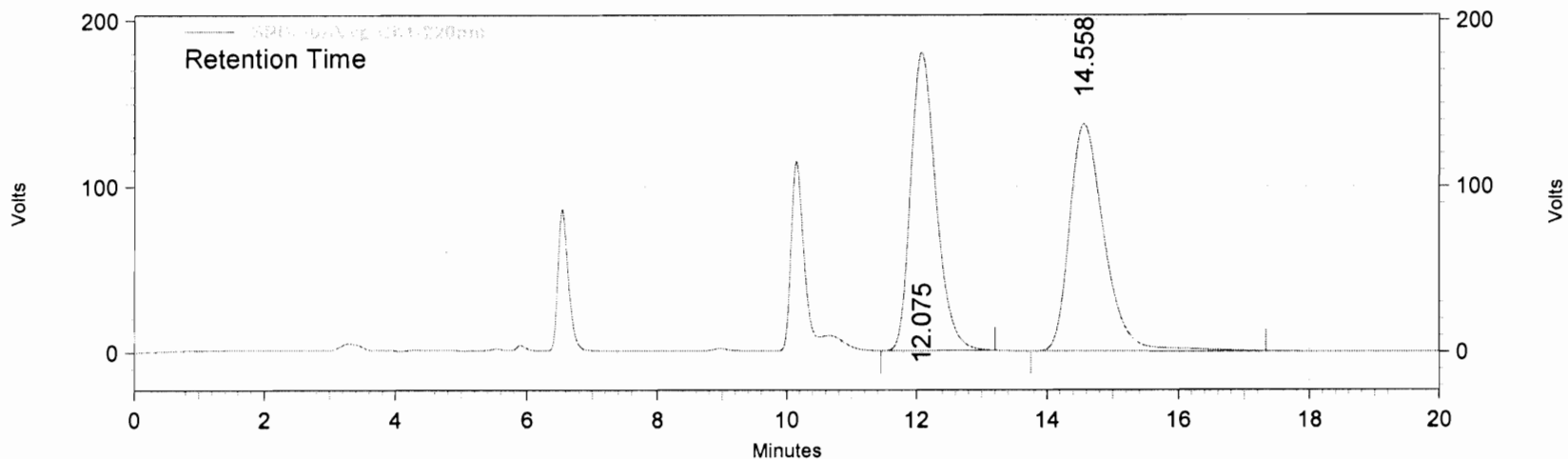

SPD-10AVvp

Ch1-220nm

Results

Retention Time

12.075

Area

Area \%

Height

Height \%

14.558

4875704

49.26

179761

56.75

50.74

137001

43.25

\begin{tabular}{|r|r|r|r|r|}
\hline Totals & 9897908 & 100.00 & 316762 & 100.00 \\
\hline
\end{tabular}<smiles>CCOP(=O)(OCC)C(O)(C[N+](=O)[O-])c1cccs1</smiles>

(5o, Racemic) 


\section{Area \% Report}

Page 1 of 1

Method Name: C:LEZStart|Projects $\backslash$ Default $\backslash$ Methods $\backslash A S$. met

Data: C:LEZStart 1 Projects Default|DatalI-TM-51OJ

User: $\quad$ System

Acquired: $\quad$ 11/18/2006 11:12:45 AM

Printed: $\quad$ 12/13/2006 11:03:34 AM

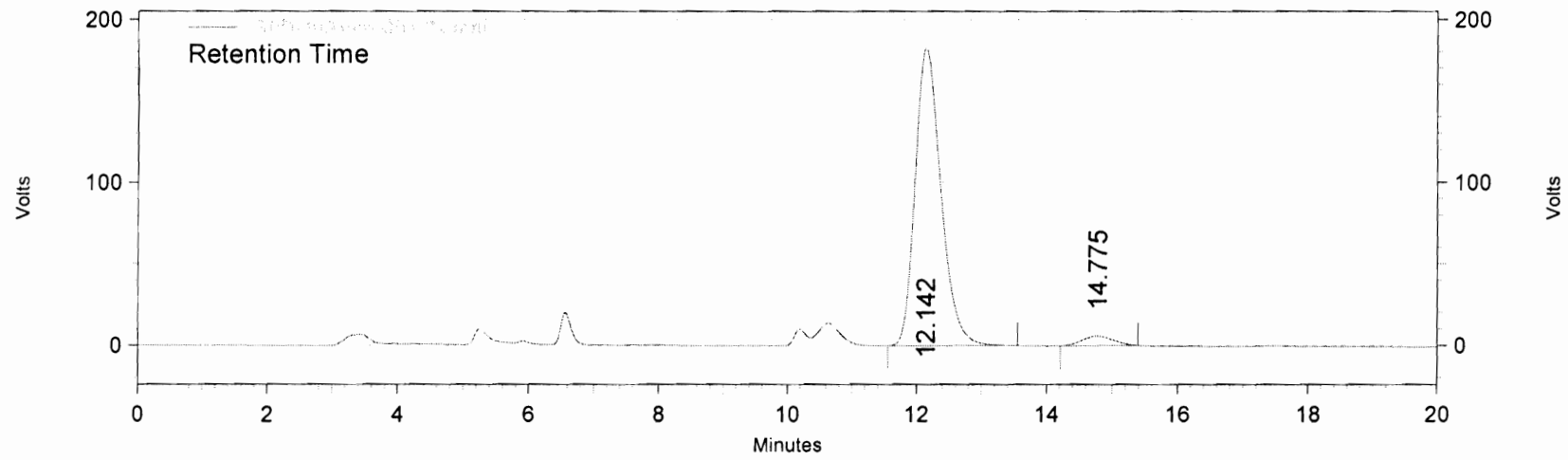

SPD-10AVyp

Ch1-220nm

Results

\begin{tabular}{rrrrrr} 
Retention Time & Area & Area \% & Height & Height \% \\
\hline 12.142 & 5055984 & 96.31 & $\begin{array}{r}182491 \\
5825\end{array}$ & $\begin{array}{r}96.91 \\
3.09\end{array}$ \\
14.775 & 193465 & 3.69 & & \\
\hline Totals & 5249449 & 100.00 & 188316 & 100.00 \\
\hline
\end{tabular}

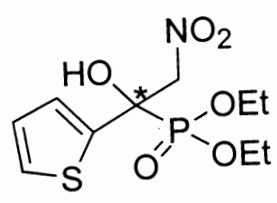

(5o, $93 \%$ ee) 


\section{Area \% Report}

Method Name: C: $\backslash$ EZStart $\backslash$ Projects $\backslash$ Default $\backslash$ Methods $\backslash$ AS.met

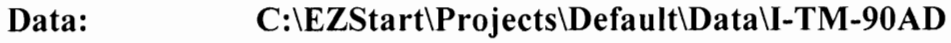

User: $\quad$ System

Acquired: $\quad 11 / 28 / 2006$ 9:52:24 AM

Printed: $\quad 12 / 13 / 200611: 03: 54$ AM

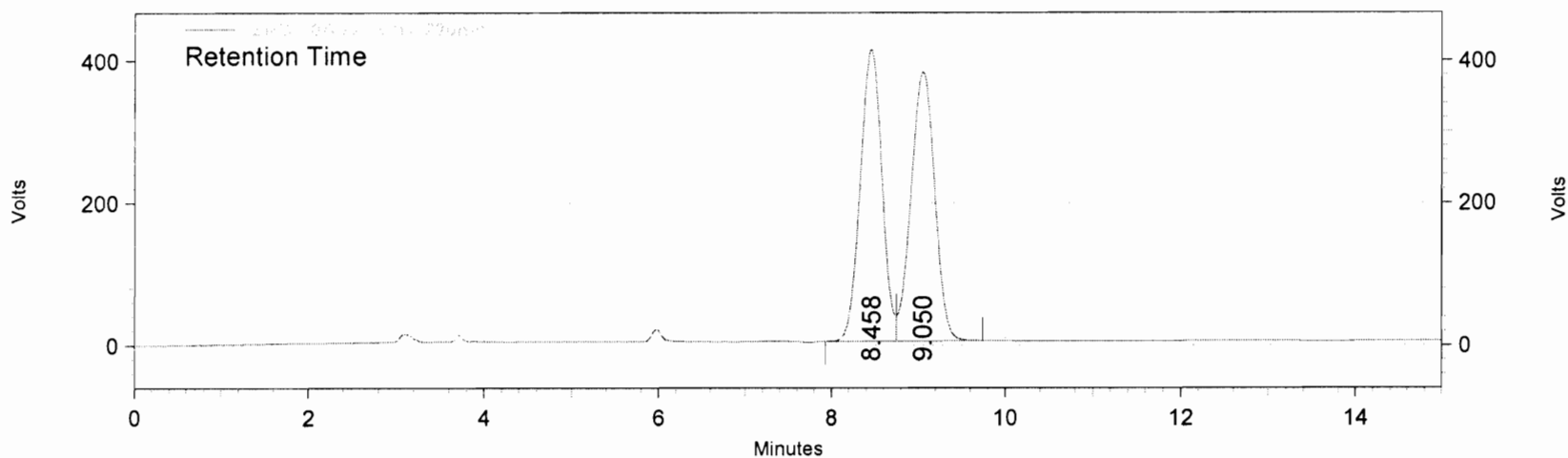

SPD-10AVvp

Ch1-220nm

Results

Retention Time

8.458

Area

Area \% Height

Height \%

9.050

7151893

50.15

377640

52.01

47.99

\begin{tabular}{|r|r|r|r|r|}
\hline Totals & 14262288 & 100.00 & 786956 & 100.00 \\
\hline
\end{tabular}<smiles>CCOP(=O)(OCC)C(C)(O)C[N+](=O)[O-]</smiles>

(5p, Racemic) 


\section{Area \% Report}

Method Name: C: $\backslash$ EZStart $\backslash$ Projects $\backslash$ Default $\backslash$ Methods $\backslash$ AS.met

Data: C: CEStart $\backslash$ Projects $\backslash$ Default $\backslash$ Data $\backslash I-T M-91 A D 5$

User: $\quad$ System

Acquired: $\quad 11 / 28 / 2006$ 10:33:14 AM

Printed: $\quad 12 / 13 / 2006$ 11:04:41 AM

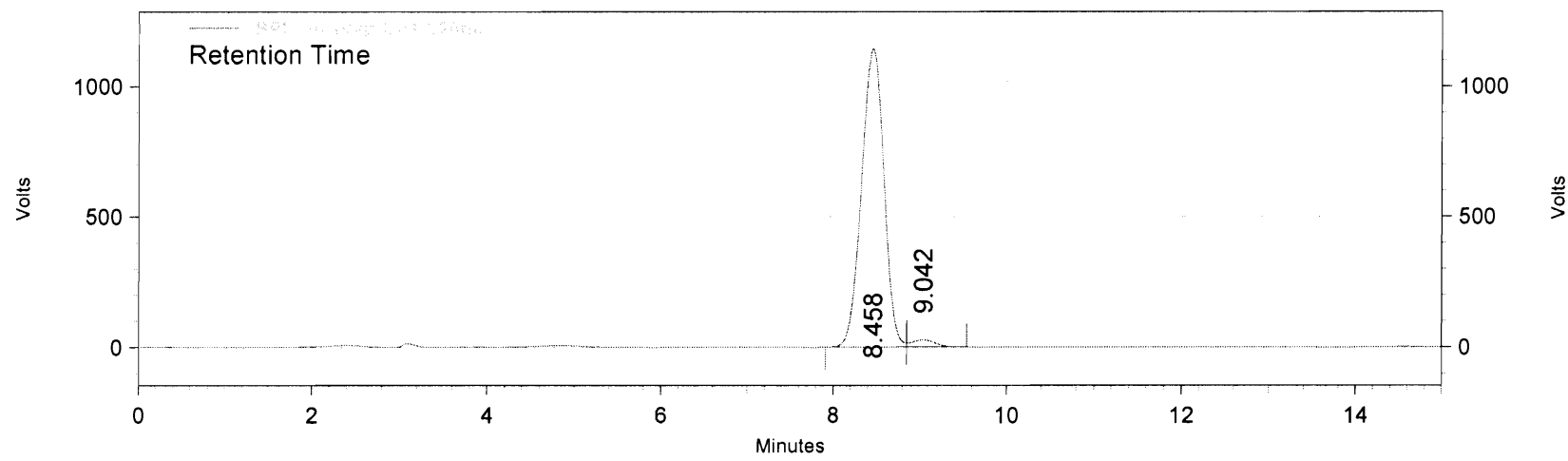

SPD-10AVvp

Ch1-220nm

Results

\begin{tabular}{rrrrrr} 
Retention Time & Area & Area \% & Height & Height \% \\
\hline 8.458 & 20599117 & 97.63 & 1143631 & 97.72 \\
9.042 & 500088 & 2.37 & 26643 & 2.28 \\
\hline Totals & 21099205 & 100.00 & 1170274 & 100.00 \\
\hline
\end{tabular}<smiles>CCOP(=O)(OCC)C(C)(O)C[N+](=O)[O-]</smiles>

$(5 p, 95 \%$ ee)

catalyst 2 


\section{Area \% Report}

Method Name: C: $\backslash$ EZStart $\backslash$ Projects $\backslash$ Default $\backslash$ Methods $\backslash$ AS.met

Data: $\quad C: \backslash$ EZStart $\backslash$ Projects $\backslash$ Default $\backslash$ Data $\backslash$ sgo

User: System

Acquired: $\quad$ 11/19/2006 3:09:34 PM

Printed: $\quad$ 12/13/2006 11:05:37 AM

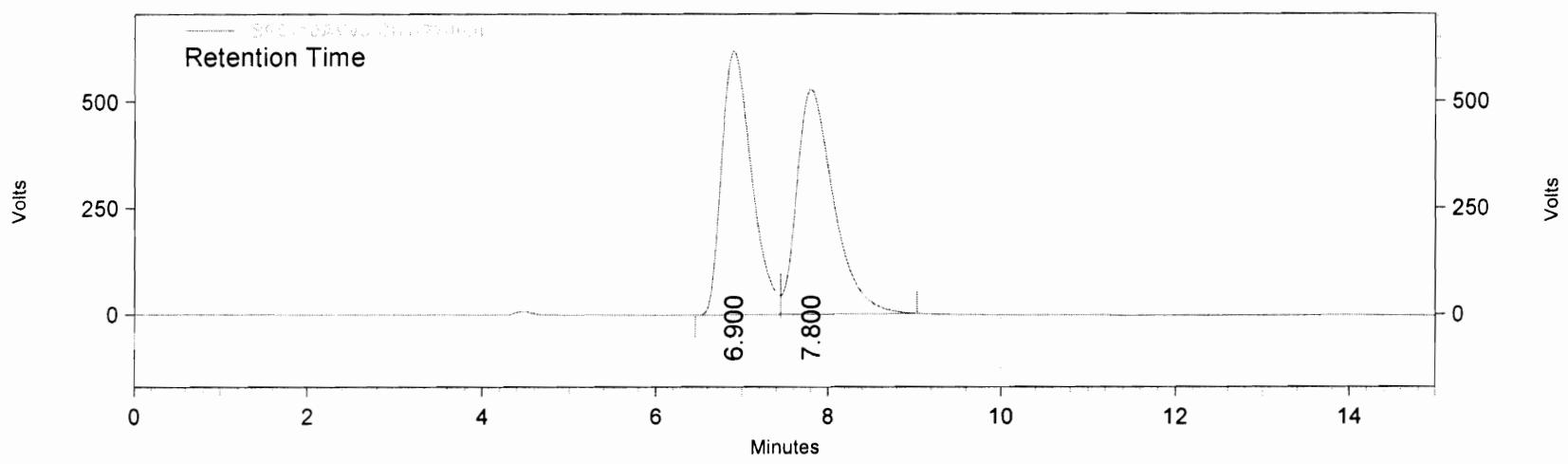

SPD-10AVvp

Ch1-220nm

Results

Retention Time

Area

Area \%

Height

619875

Height \%

6.900

15035017

48.89

527595

54.02

7.800

15715724

51.11

45.98

Totals

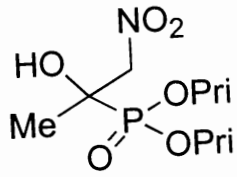

(5q, Racemic) 


\section{Area \% Report}

Method Name: C: $\backslash$ EZStart $\backslash$ Projects $\backslash$ Default $\backslash$ Methods $\backslash$ AS.met

Data: $\quad C: \backslash E Z S t a r t \backslash P r o j e c t s \backslash D e f a u l t \backslash D a t a \backslash I-T M-46 A S$

User: $\quad$ System

Acquired: $\quad$ 11/19/2006 3:40:47 PM

Printed: $\quad 12 / 13 / 2006$ 11:05:20 AM

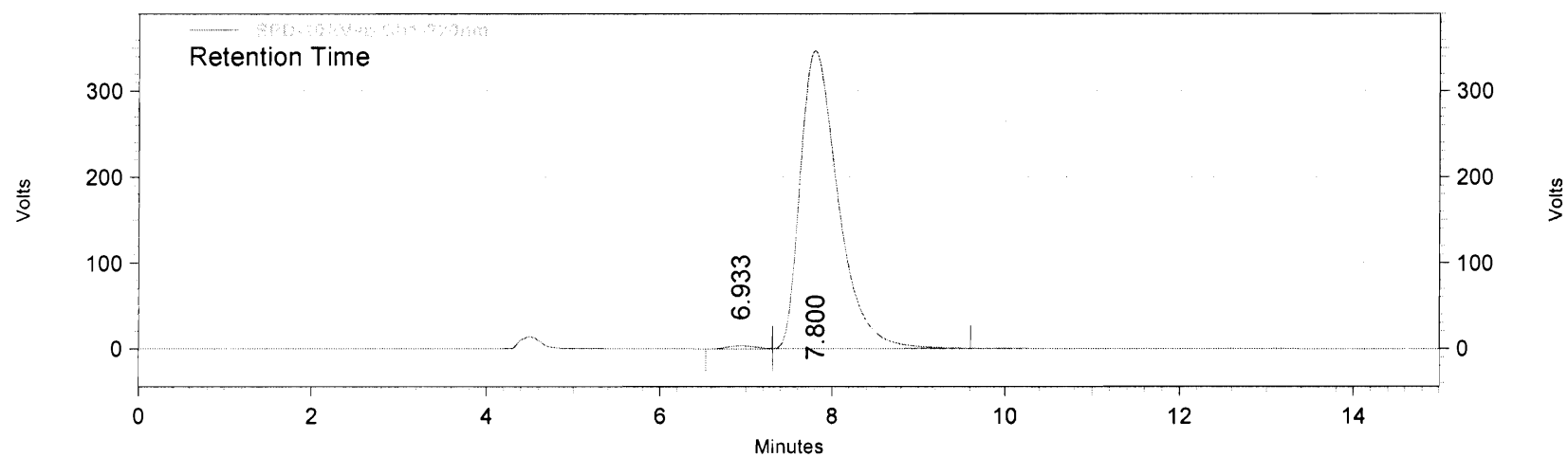

SPD-10AVvp

Ch1-220nm

Results

Retention Time Area

Area $\%$ Height

Height \%

6.933

82747

0.77

3464 0.99

7.800

10634250

99.23

347036

99.01

\begin{tabular}{|r|r|r|r|r|}
\hline Totals & 10716997 & 100.00 & 350500 & 100.00 \\
\hline
\end{tabular}<smiles>CCCOP(=O)(OCCC)C(C)(O)C[N+](=O)[O-]</smiles>

(5q, $98 \%$ ee)

catalyst 2 


\section{Area \% Report}

Method Name: C: $\backslash$ EZStart $\backslash$ Projects $\backslash$ Default $\backslash$ Methods $\backslash$ AS. met

Data: $\quad C: \backslash$ EZStart $\backslash$ Projects $\backslash$ Default $\backslash$ Data $\backslash I-T M-75 S$

User: $\quad$ System

Acquired: $\quad$ 11/22/2006 12:31:59 PM

Printed: $\quad 12 / 13 / 2006$ 11:06:02 AM

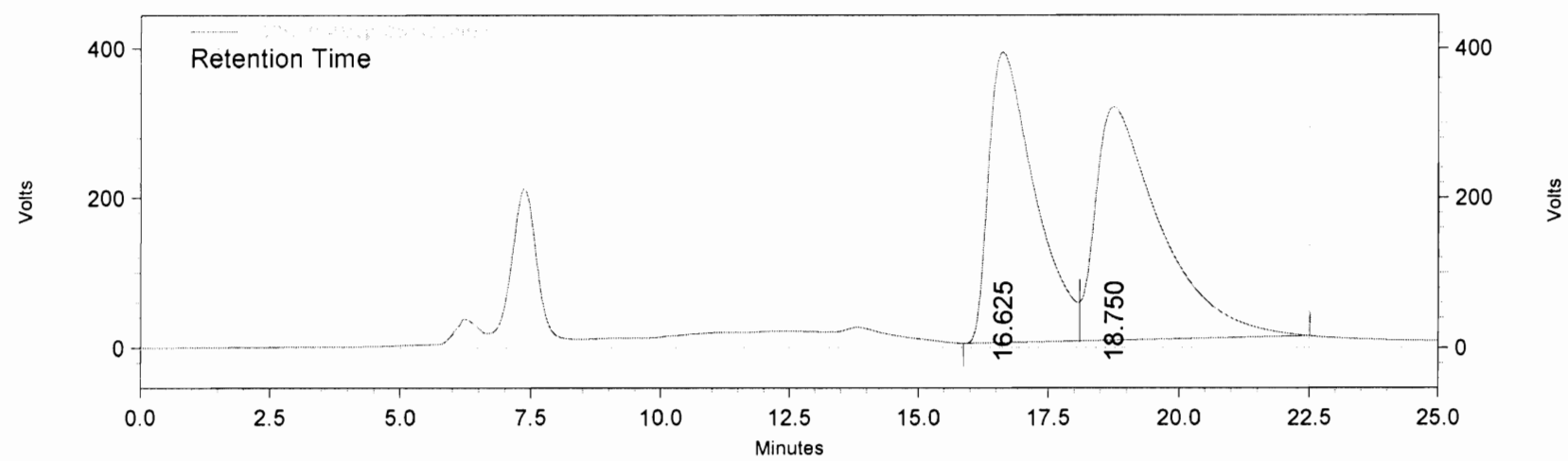

SPD-10AVvp

Ch1-220nm

Results

Retention Time

Area

Area \%

46.90

Height

16.625

23890790

53.10

387897

Height $\%$

18.750

27049306

311261

44.52

\begin{tabular}{|r|r|r|r|r|}
\hline Totals & 50940096 & 100.00 & 699158 & 100.00 \\
\hline
\end{tabular}<smiles>CCOP(=O)(OCC)C(O)(CC)C[N+](=O)[O-]</smiles>

(5r, Racemic) 


\title{
Area \% Report
}

\author{
Method Name: C:|EZStart $\backslash$ Projects $\backslash$ Default $\backslash$ Methods $\backslash A S$.met \\ Data: C: $\backslash$ EZStart $\backslash$ Projects $\backslash$ Default $\backslash$ Data $\backslash I-T M-76$ \\ User: $\quad$ System \\ Acquired: $\quad$ 11/22/2006 9:59:15 AM \\ Printed: $\quad$ 12/13/2006 11:06:20 AM
}

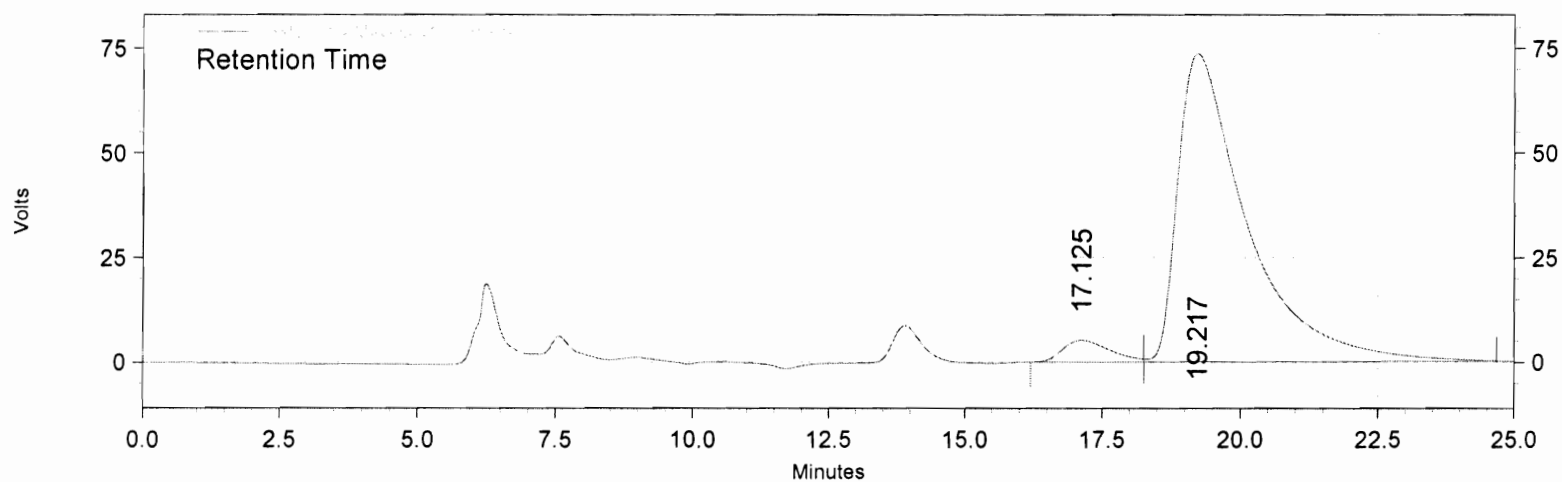

SPD-10AVvp

Ch1-220nm

Results

\begin{tabular}{rrrrrr|} 
Retention Time & Area & Area \% & Height & Height \% \\
\hline 17.125 & 301373 & 4.51 & 5118 & 6.50 \\
19.217 & 6383663 & 95.49 & 73566 & 93.50 \\
\hline \multicolumn{2}{|c|}{ Totals } & 6685036 & 100.00 & 78684 & 100.00 \\
\hline
\end{tabular}

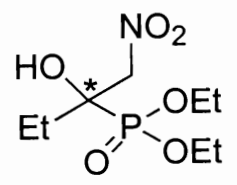

(5r, $91 \%$ ee)

catalyst 2 


\section{Area \% Report}

Method Name: C: $\backslash$ EZStart $\backslash$ Projects $\backslash$ Default $\backslash$ Methods $\backslash$ AS.met

Data: C: $\backslash$ EZStart $\backslash$ Projects $\backslash$ Default $\backslash$ Data $\backslash I-T M-79 P$

User: $\quad$ System

Acquired: $\quad$ 11/23/2006 2:10:06 PM

Printed: $\quad 12 / 13 / 2006$ 11:07:09 AM

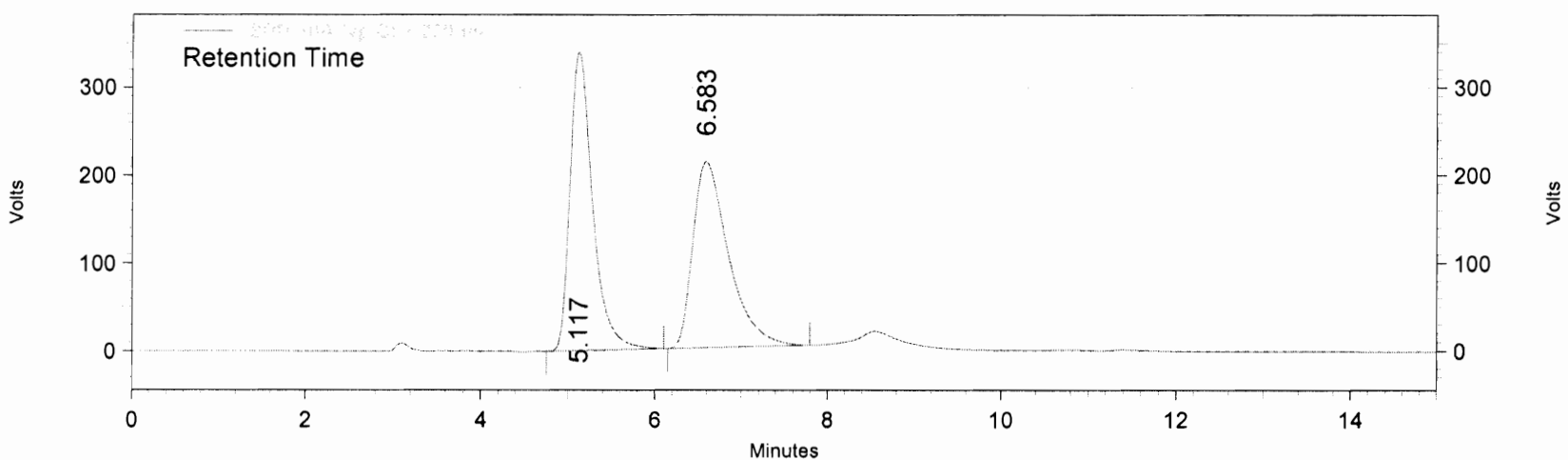

SPD-10AVvp

Ch1-220nm

Results

Retention Time

Area

Area \%

50.23

Height

339593

Height \%

6.583

6077425

49.77

211898

61.58

38.42

\begin{tabular}{|r|r|r|r|r|}
\hline Totals & 12211376 & 100.00 & 551491 & 100.00 \\
\hline
\end{tabular}

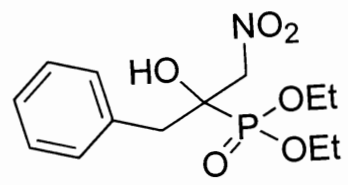

(5s, Racemic) 


\section{Area \% Report}

Method Name: C:\EZStart $\backslash$ Projects $\backslash$ Default $\backslash$ Methods $\backslash$ AS.met

Data: C: $\quad$ EZStart $\backslash$ Projects $\backslash$ Default $\backslash$ Data $\backslash I-T M-80 A S$

User: $\quad$ System

Acquired: $\quad$ 11/23/2006 4:15:52 PM

Printed: $\quad 12 / 13 / 2006$ 11:07:27 AM

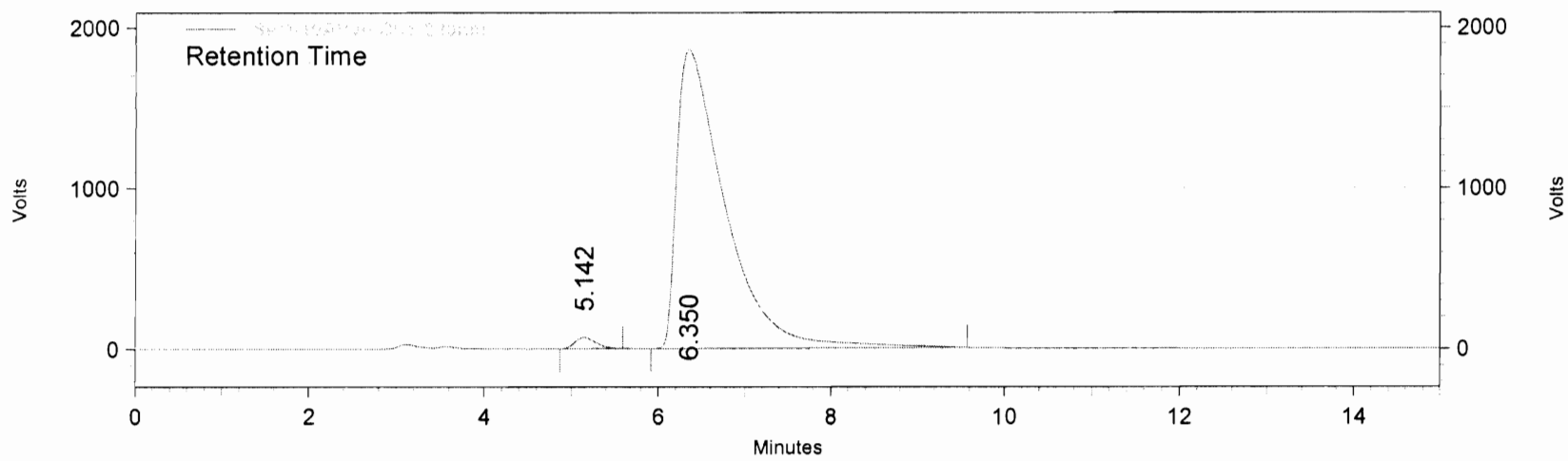

SPD-10AVvp

Ch1-220nm

Results

Retention Time

Area

Area \%

Height

Height \%

5.142

1133174

1.54

68250

3.54

6.350

72226910

98.46

1858362

96.46

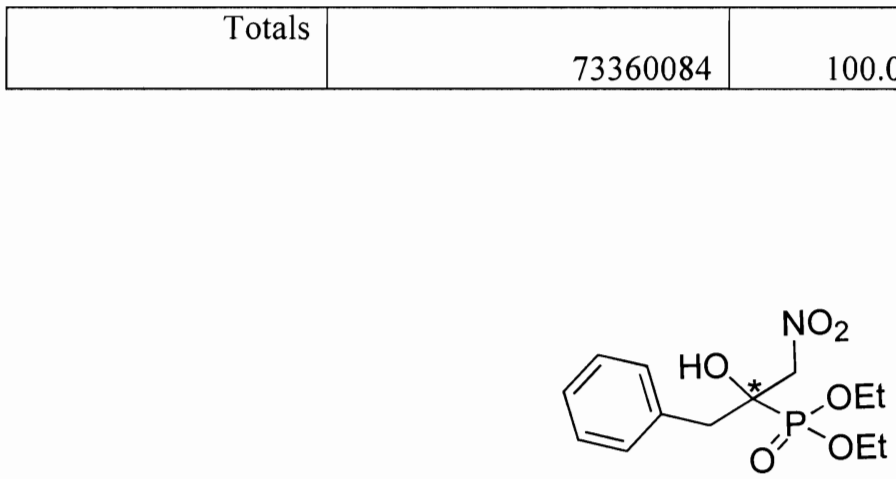

(5s, $97 \%$ ee)

catalyst 2 


\section{Area \% Report}

Method Name: C: $\backslash$ EZStart $\backslash$ Projects $\backslash$ Default $\backslash$ Methods $\backslash$ AS.met

Data: C: $\quad$ EZStart $\backslash$ Projects $\backslash$ Default $\backslash$ Data $\backslash$ I-TM-82OJ

User: System

Acquired: $\quad$ 11/24/2006 1:19:45 PM

Printed: $\quad 12 / 13 / 2006$ 11:07:51 AM

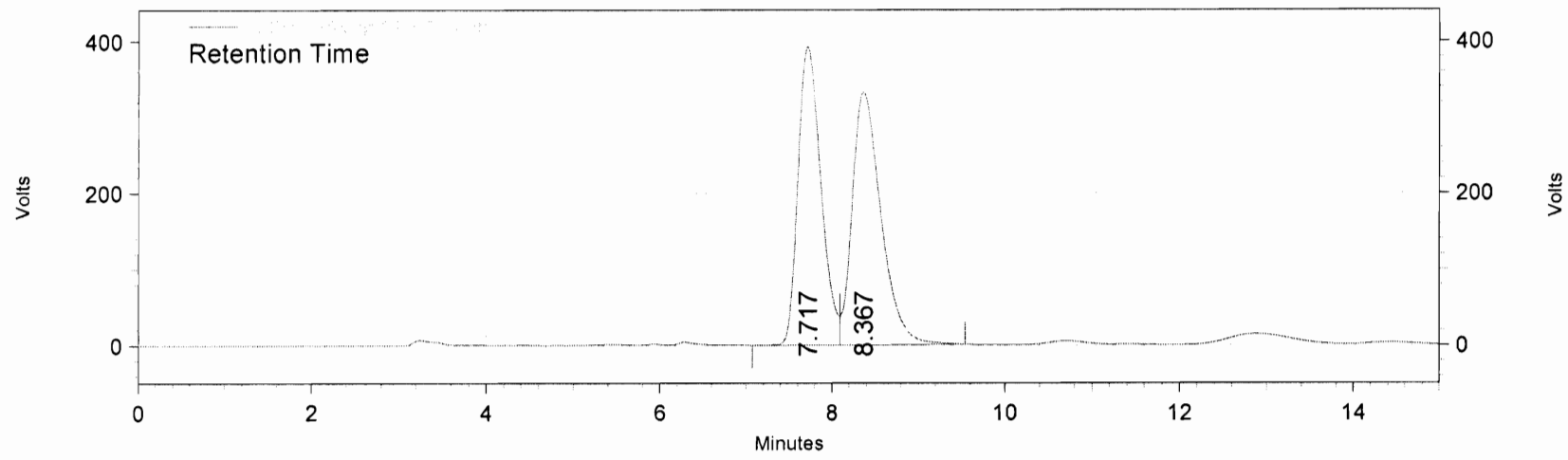

SPD-10AVvp

Ch1-220nm

Results

Retention Time

7.717

Area

Area \%

48.55

Height

391955

Height \%

8.367

7692932

51.45

332089

45.87

\begin{tabular}{|r|r|r|r|r|}
\hline Totals & 14951682 & 100.00 & 724044 & 100.00 \\
\hline
\end{tabular}<smiles>CCOP(=O)(OCC)C(O)(CCc1ccccc1)[N+](=O)[O-]</smiles>

(5t, Racemic) 


\section{Area \% Report}

Method Name: C: $\backslash$ EZStart $\backslash$ Projects $\backslash$ Default $\backslash$ Methods $\backslash A S$.met

Data: C: $\quad$ EZStart $\backslash$ Projects $\backslash$ Default $\backslash$ Data $\backslash$ I-TM-117OJ

User: System

Acquired: $\quad$ 12/7/2006 12:51:11 PM

Printed: $\quad 12 / 13 / 2006$ 10:31:27 AM

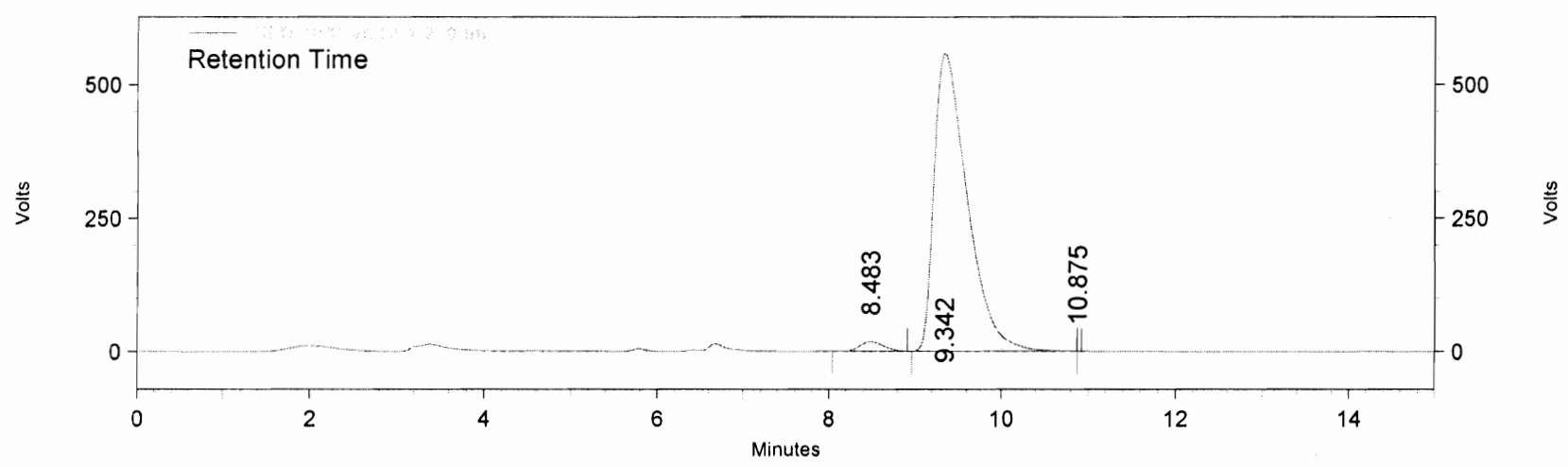

SPD-10AVvp

Ch1-220nm

Results

Retention Time

8.483

Area

Area \%

Height

Height \%

9.342

336449

2.21

17858

3.10

10.875

14872465

97.79

558277

96.88

Totals

174

117

0.02

\begin{tabular}{|r|r|r|r|r|}
\hline Totals & 15209088 & 100.00 & 576252 & 100.00 \\
\hline
\end{tabular}<smiles>CCOP(=O)(OCC)C(O)(CCc1ccccc1)[N+](=O)[O-]</smiles>

(5t, $96 \%$ ee)

catalyst 3 


\section{Area \% Report}

Method Name: C: $\backslash$ EZStart $\backslash$ Projects $\backslash$ Default $\backslash$ Methods $\backslash$ AS. met

Data: C: $\backslash$ EZStart $\backslash$ Projects $\backslash$ Default $\backslash$ Data $\backslash I-T M-126 R$

User: System

Acquired: $\quad$ 12/9/2006 1:29:22 PM

Printed: $\quad 12 / 13 / 2006$ 10:34:24 AM

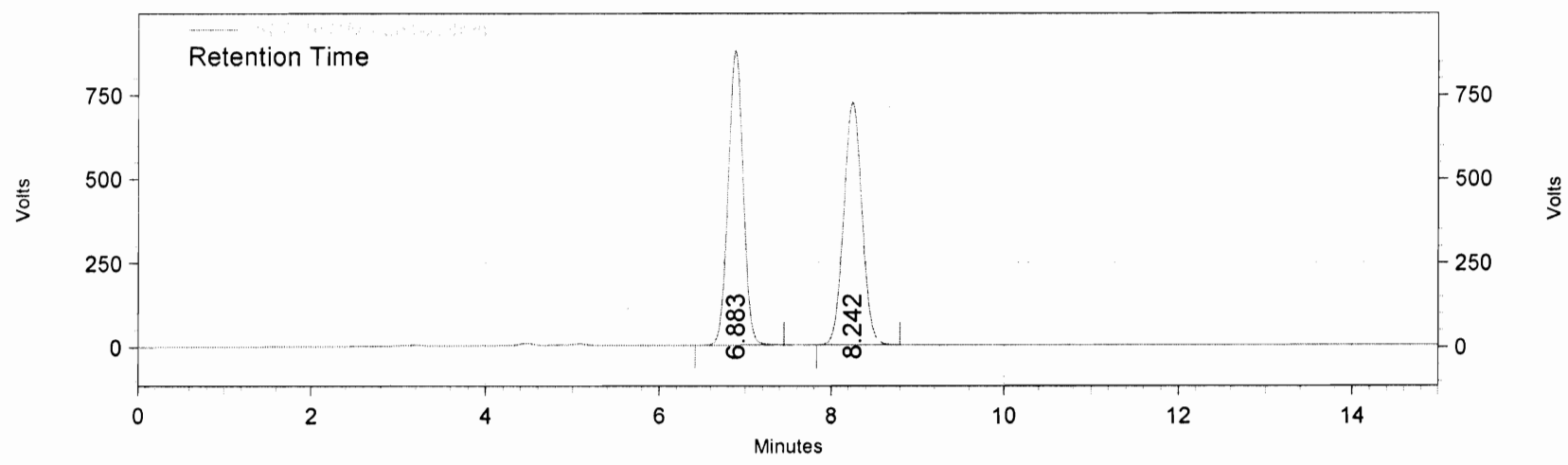

SPD-10AVvp

Ch1-220nm

Results

Retention Time

Area

Area $\%$

Height

Height \%

6.883

10617446

50.05

877564

49.95

722132

54.86

8.242

10595901

100.00

1599696

100.00

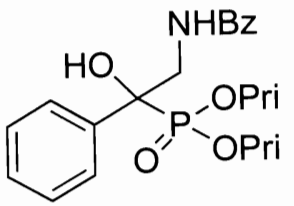

(6, Racemic) 


\section{Area \% Report}

Method Name: C:|EZStart|Projects|Default|Methods\AS.met

Data: C:IEZStart|Projects|Default|DatalI-TM-142AD

User: $\quad$ System

Acquired: $\quad 12 / 13 / 2006$ 10:12:06 AM

Printed: $\quad$ 12/13/2006 12:53:13 PM

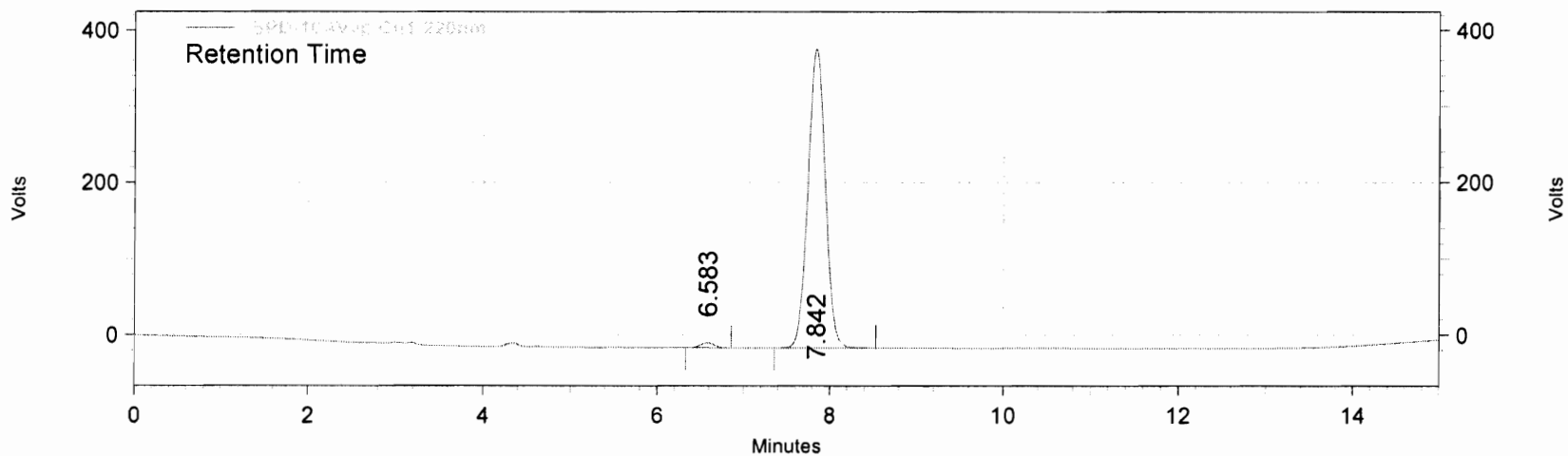

SPD-10AVvp

Ch1-220nm

Results

\begin{tabular}{rrrrrr|} 
Retention Time & Area & Area \% & Height & Height \% \\
\hline 6.583 & 72861 & 1.31 & 6532 & 1.64 \\
7.842 & 5498925 & 98.69 & 392765 & 98.36 \\
\hline Totals & 5571786 & 100.00 & 399297 & 100.00 \\
\hline
\end{tabular}<smiles>CCCOP(=O)(OC(C)C)C(O)(CNC(=O)c1ccccc1)c1ccccc1</smiles>

$(6,97 \%$ ee $)$ 